\title{
On the classification of quasitoric manifolds over dual cyclic polytopes
}

\author{
SHO HASUI
}

\begin{abstract}
For a simple $n$-polytope $P$, a quasitoric manifold over $P$ is a $2 n$-dimensional smooth manifold with a locally standard action of an $n$-dimensional torus for which the orbit space is identified with $P$. This paper acheives the topological classification of quasitoric manifolds over the dual cyclic polytope $C^{n}(m)^{*}$ when $n>3$ or $m-n=$ 3. Additionally, we classify small covers, the "real version" of quasitoric manifolds, over all dual cyclic polytopes.
\end{abstract}

57R19, 57S25

\section{Introduction}

Quasitoric manifolds were introduced by Davis and Januszkiewicz [5] as a topological generalization of nonsingular projective toric varieties. More precisely, a quasitoric manifold is a $2 n$-dimensional manifold $M$ with a locally standard $T^{n}$-action for which the orbit space $M / T^{n}$ is identified with a simple polytope as a manifold with corners. Here "locally standard" means that the $T^{n}$-action is locally identified with the coordinate-wise multiplication of $T^{n}$ on $\mathbb{C}^{n}$, where $T^{n}$ is regarded as $T^{n}=\left\{\left(z_{1}, \ldots, z_{n}\right) \in \mathbb{C}^{n}|| z_{i} \mid=1, i=1, \ldots, n\right\}$. The simplest example is $\mathbb{C P}^{n}$ with the standard $T^{n}$-action, whose supporting simple polytope is the $n$-dimensional simplex $\Delta^{n}$.

There are other examples of quasitoric manifolds in Orlik and Raymond [7], where it was shown that many compact oriented one-connected four-manifolds with smooth effective $T^{2}$-actions are quasitoric manifolds. In fact, that paper also provides a classification of four-dimensional quasitoric manifolds up to diffeomorphism.

We consider the classification of quasitoric manifolds over the dual of a cyclic polytope up to (i) weakly equivariant homeomorphism, (ii) homeomorphism and (iii) cohomology equivalence. Here a "cohomology equivalence" between two topological spaces means there exists a graded ring isomorphism between their integral cohomology rings that is not necessarily induced by a continuous map. For a group $G$, a map $f$ between two $G$-spaces is called weakly equivariant if the condition $f(g \cdot x)=\psi(g) \cdot f(x)$ holds 
for some automorphism $\psi$ of $G$, where $x$ and $g$ are arbitrary points in the domain and $G$ respectively.

In [5], Davis and Januszkiewicz showed that every quasitoric manifold is represented by a pair $(P, \lambda)$, where $P$ is a simple polytope and $\lambda$ is a characteristic matrix on $P$ (see Definition 2.5), up to their equivalence (which is stronger than weakly equivariant homeomorphism). The next section is devoted to a review of their construction and the classification of quasitoric manifolds up to weakly equivariant homeomorphism (Proposition 2.12).

There are some results for the classification of quasitoric manifolds up to homeomorphism. The classification of quasitoric manifolds over $\Delta^{n}$ is trivial by construction; see [5, Example 1.18]. Using the results of Orlik and Raymond [7], we obtain the classification over convex polygons; see [5, Example 1.20]. Moreover, Choi, Park and Suh obtained in [3] the topological classification of quasitoric manifolds over the product of two simplices $\Delta^{n} \times \Delta^{m}$.

For a simple polytope $P$, let us denote by $\mathcal{M}_{P}$ the set of all weakly equivariant homeomorphism classes of quasitoric manifolds over $P$. Similarly, let $\mathcal{M}_{P}^{\text {homeo }}$ denote the set of all homeomorphism classes and $\mathcal{M}_{P}^{\text {coh }}$ denote the set of all cohomology equivalence classes. Then we have a sequence of surjections $\mathcal{M}_{P} \rightarrow \mathcal{M}_{P}^{\text {homeo }} \rightarrow \mathcal{M}_{P}^{\text {coh }}$. Let us summarize the classification results using this notation.

Theorem 1.1 [3; 5] For quasitoric manifolds over a simplex, a convex polygon and the product of two simplices, we have the following classification. Note that $\Delta^{1} \times \Delta^{1}$ is equal to the convex tetragon $P_{4}$.

(i) For the $n$-dimensional simplex $\Delta^{n}, \# \mathcal{M}_{\Delta^{n}}=\# \mathcal{M}_{\Delta^{n}}^{\text {homeo }}=\# \mathcal{M}_{\Delta^{n}}^{\text {coh }}=1$.

(ii) For the convex $m$-gon $P_{m}$, where $m \geq 4, \mathcal{M}_{P_{m}}$ has countably infinite elements and

$$
\# \mathcal{M}_{P_{m}}^{\text {homeo }}=\# \mathcal{M}_{P_{m}}^{\text {coh }}= \begin{cases}\frac{1}{2}(m-1) & \text { if } m \text { is odd, } \\ \frac{1}{2} m+1 & \text { if } m \text { is even. }\end{cases}
$$

(iii) For $\Delta^{n} \times \Delta^{m}$, where $n \geq 1$ and $m \geq 2, \mathcal{M}_{\Delta^{n} \times \Delta^{m}}^{\text {coh }}$ has countably infinite elements, and $\mathcal{M}_{\Delta^{n} \times \Delta^{m}} \rightarrow \mathcal{M}_{\Delta^{n} \times \Delta^{m}}^{\text {homeo }}$ is not bijective but $\mathcal{M}_{\Delta^{n} \times \Delta^{m}}^{\text {homeo }} \rightarrow \mathcal{M}_{\Delta^{n} \times \Delta^{m}}^{\text {coh }}$ is.

Corollaries 2.16 and 4.7 give representatives of the elements of $\mathcal{M}_{\Delta^{n}}$ and $\mathcal{M}_{P_{m}}^{\text {homeo }}$ respectively.

We will show the following new results for the classification of quasitoric manifolds over the dual cyclic polytope $C^{n}(m)^{*}$. Note that in the case where $n \leq 2$ or $m-n \leq 2$, the topological classification of quasitoric manifolds is already known since $C^{2}(m)^{*}$ 
is the convex $m$-gon and $C^{n}(n+2)^{*}$ is $\Delta^{l} \times \Delta^{n-l}$, where $l$ denotes the largest integer less than $\frac{1}{2} n$.

Theorem 1.2 If $n \geq 4$ and $m-n \geq 4$, or $n \geq 6$ and $m-n \geq 3$, there exist no quasitoric manifolds over $C^{n}(m)^{*}$.

Theorem 1.3 For quasitoric manifolds over the dual cyclic polytope $C^{n}(n+3)^{*}$, we have the following classification:

(i) The map $\mathcal{M}_{C^{3}(6)^{*}} \rightarrow \mathcal{M}_{C^{3}(6)^{*}}^{\text {homeo }}$ is not bijective but $\mathcal{M}_{C^{3}(6)^{*}}^{\text {homeo }} \rightarrow \mathcal{M}_{C^{3}(6)^{*}}^{\text {ch }}$ is, and all the classes have countably infinite elements.

(ii) $\# \mathcal{M}_{C^{4}(7)^{*}}=\# \mathcal{M}_{C^{4}(7)^{*}}^{\text {homeo }}=\# \mathcal{M}_{C^{4}(7)^{*}}^{\text {coh }}=4$.

(iii) $\# \mathcal{M}_{C^{5}(8)^{*}}=\# \mathcal{M}_{C^{5}(8)^{*}}^{\text {homeo }}=\# \mathcal{M}_{C^{5}(8)^{*}}^{\text {coh }}=46$.

Note that the classification of quasitoric manifolds over $C^{3}(m)^{*}$ for $m \geq 7$ is still open since it is quite complicated, and it remains a subject for further study.

Theorem 1.3 yields a partial affirmative answer to the cohomological rigidity problem for quasitoric manifolds over dual cyclic polytopes. A class of topological spaces is called cohomologically rigid (for homeomorphisms) if two spaces in the class are homeomorphic if and only if their cohomology rings (with coefficients in an appropriate ring) are isomorphic as graded rings. Note that the Betti numbers of a quasitoric manifold determine the $f$-vector of the supporting simple polytope (see Definition 2.19 and Theorem 2.20). As a corollary of Theorem 1.1, we obtain cohomological rigidity of quasitoric manifolds over the simplices, the convex polygons and the products of two simplices. There are several other results on the cohomological rigidity of quasitoric manifolds. For more information, we refer the reader to Choi, Masuda and Suh [2].

Replacing $T$ by $\mathbb{Z} / 2$ and $\mathbb{C}$ by $\mathbb{R}$ in the definition of a quasitoric manifold, we obtain the notion of a small cover. Any quasitoric manifold has an involution called "conjugation" for which the fixed point set is homeomorphic to a small cover. The lifting problem asks whether or not a small cover can be realized as the fixed point set for the involution on a quasitoric manifold. The answer is affirmative for any small cover of dimension $\leq 3$ (Proposition 2.18).

For a simple polytope $P$, let us denote by $\mathbb{R} \mathcal{M}_{P}$ the set of all weakly equivariant homeomorphism classes of small covers over $P$. Similarly, let $\mathbb{R}_{\mathcal{P}} \mathcal{M}_{P}^{\text {homeo }}$ denote the set of all homeomorphism classes and $\mathbb{R}_{\mathbb{P}} \mathcal{M}_{P}^{\text {coh }}$ denote the set of all mod 2 cohomology equivalence classes. We will show the following theorems. 
Theorem 1.4 If $n \geq 4$ and $m-n \geq 4$, or $n \geq 6$ and $m-n \geq 3$, there exists no small cover over $C^{n}(m)^{*}$.

Theorem 1.5 For small covers over the dual cyclic polytope $C^{n}(m)^{*}$, we have the following classification:

(i) $\mathbb{R}_{C^{3}(m)^{*}} \rightarrow \mathbb{R}_{C^{3}(m)^{*}}^{\text {homeo }}$ is not bijective but $\mathbb{R}_{C^{3}(m)^{*}} \rightarrow \mathbb{R}_{C^{3}(m)^{*}}^{\text {homeo }}$ is.

(ii) $\#_{\mathbb{R}} \mathcal{M}_{C^{4}(7)^{*}}=\#_{\mathbb{R}} \mathcal{M}_{C^{4}(7)^{*}}^{\text {homeo }}=\#_{\mathbb{R}} \mathcal{M}_{C^{4}(7)^{*}}^{\text {coh }}=1$.

(iii) $\#_{\mathbb{R}} \mathcal{M}_{C^{5}(8)^{*}}=\#_{\mathbb{R}} \mathcal{M}_{C^{5}(8)^{*}}^{\text {homeo }}=\#_{\mathbb{R}} \mathcal{M}_{C^{5}(8)^{*}}^{\text {coh }}=1$.

Note that Theorem 1.2 is a corollary of Theorem 1.4. Indeed, if there exists a quasitoric manifold over a simple polytope $P$, the fixed point set for the conjugation involution provides a small cover over the same polytope $P$.

With Theorems 1.3 and 1.5, we obtain the following corollary.

Corollary 1.6 The answer to the lifting problem is affirmative for all small covers over dual cyclic polytopes.

Now we briefly describe how this paper is organized. In Section 2, we recall some basic constructions and properties of quasitoric manifolds and small covers. In Section 3, we recall some properties of a cyclic polytope, introducing some notation and the connected sum operation. Using the connected sum operation, in Section 4 we give a "purely quasitoric" proof for the topological classification of quasitoric manifolds and small covers over $C^{2}(m)^{*}$. In Section 5 , we classify quasitoric manifolds over $C^{3}(6)^{*}$ and small covers over $C^{3}(m)^{*}$, again using the connected sum operation. In Section 6, we prove Theorem 1.4 and list all small covers over $C^{4}(7)^{*}$ and $C^{5}(8)^{*}$. In Section 7, we list all elements of $\mathcal{M}_{C^{4}(7)^{*}}$ and show that their integer cohomology rings are not isomorphic to each other. In Section 8, we similarly show the classification for $C^{5}(8)^{*}$.

\section{Basic constructions and properties}

The purpose of this section is to define a quasitoric manifold and describe some of its properties. Let us start by recalling some basic concepts and constructions for convex polytopes. 


\subsection{Polytopes}

An $n$-dimensional convex polytope is called simple if exactly $n$ facets meet at each vertex. We can naturally regard a simple polytope as a manifold with corners, in the following sense: an $n$-dimensional manifold with corners is a Hausdorff space that has an atlas of open subsets and homeomorphisms from them to open subsets of $\mathbb{R}_{+}^{n}=\left\{\left(x_{1}, \ldots, x_{n}\right) \in \mathbb{R}^{n} \mid x_{i} \geq 0, i=1, \ldots, n\right\}$, such that the transition maps preserve the canonical stratification of $\mathbb{R}_{+}^{n}$.

Let $P$ be an $n$-dimensional simple polytope with $m$ facets which are labeled by $F_{1}, \ldots, F_{m}$. We define an abstract simplicial complex $K_{P}$ on $\{1, \ldots, m\}$ by

$$
\left\{i_{1}, \ldots, i_{k}\right\} \in K_{P} \Longleftrightarrow F_{i_{1}} \cap \cdots \cap F_{i_{k}} \neq \varnothing .
$$

$K_{P}$ is identified with the face poset of $P^{*}$. Note that $\left\{i_{1}, \ldots, i_{n}\right\}$ is a maximal face of $K_{P}$ if and only if $F_{i_{1}}, \ldots, F_{i_{n}}$ meet at a vertex.

The nerve of $K_{P}$ provides a triangulation of $P$. We can see this as follows: Fix a point $c_{G} \in \operatorname{rel}$ int $G$ for each face $G$, and identify a chain $G_{1} \supsetneq \cdots \supsetneq G_{k}$ with the $(k-1)-$ simplex spanned by $c_{G_{1}}, \ldots, c_{G_{k}}$. Therefore, if there is a simplicial isomorphism between $K_{P}$ and $K_{P^{\prime}}$, it induces a homeomorphism between $P$ and $P^{\prime}$. We obtain the following proposition.

Proposition 2.1 Let $P$ and $P^{\prime}$ be simple polytopes. Then a simplicial isomorphism between $K_{P}$ and $K_{P^{\prime}}$ can be realized as a homeomorphism between $P$ and $P^{\prime}$ as manifolds with corners. Conversely, a homeomorphism between $P$ and $P^{\prime}$ as manifolds with corners induces a simplicial isomorphism between $K_{P}$ and $K_{P^{\prime}}$.

\subsection{Definition of a quasitoric manifold}

To define quasitoric manifolds and consider their classification, we recall some basic concepts of $T^{n}$-manifolds.

The standard action of the $n$-dimensional torus $T^{n}=\left(S^{1}\right)^{n}$ on $\mathbb{C}^{n}$ is given as

$$
T^{n} \times \mathbb{C}^{n} \rightarrow \mathbb{C}^{n}, \quad\left(t_{1}, \ldots, t_{n}\right) \times\left(z_{1}, \ldots, z_{n}\right) \mapsto\left(t_{1} z_{1}, \ldots, t_{n} z_{n}\right),
$$

where we canonically regard $T^{n}$ as $\left\{\left(z_{1}, \ldots, z_{n}\right) \in \mathbb{C}^{n}|| z_{i} \mid=1, i=1, \ldots, n\right\}$. Note that the orbit space for this action is naturally identified with the positive cone $\mathbb{R}_{+}^{n}$.

A $T^{n}$-manifold is a differentiable manifold with a smooth action of $T^{n}$.

Definition 2.2 Let $G$ be a group, $X$ and $Y$ be $G$-spaces and $f$ be a map from $X$ to $Y$. The map $f$ is said to be weakly equivariant if the condition $f(g \cdot x)=\psi(g) \cdot f(x)$ holds for any $x \in M$ and $g \in G$, where $\psi$ is some automorphism of $G$. 
Let $M$ be a $2 n$-dimensional $T^{n}$-manifold. A standard chart on $M$ is a pair $(U, f)$, where $U$ is a $T^{n}$-stable open subset of $M$ and $f$ is a weakly equivariant diffeomorphism from $U$ onto some $T^{n}$-stable open subset of $\mathbb{C}^{n}$. An atlas which consists of standard charts is called a standard atlas. If a $2 n$-dimensional $T^{n}$-manifold has a standard atlas, then we say that the $T^{n}$-action is locally standard. The orbit space for a locally standard action is naturally regarded as a manifold with corners. Then we define a quasitoric manifold as follows.

Definition 2.3 A 2n-dimensional $T^{n}$-manifold is said to be a quasitoric manifold over a simple polytope $P$ if the action is locally standard and the orbit space is homeomorphic to $P$ as a manifold with corners.

According to Proposition 2.1, we do not have to distinguish between combinatorially equivalent simple polytopes in the above definition. The following proposition is obvious.

Proposition 2.4 Let $M$ and $M^{\prime}$ be quasitoric manifolds over simple polytopes $P$ and $P^{\prime}$ respectively. A weakly equivariant homeomorphism from $M$ to $M^{\prime}$ descends to a homeomorphism from $P$ to $P^{\prime}$ as manifolds with corners.

\subsection{Classification up to weakly equivariant homeomorphism}

The notion of a characteristic matrix plays a central role in the classification of quasitoric manifolds. Let $P$ be an $n$-dimensional simple polytope with $m$ facets $F_{1}, \ldots, F_{m}$.

Definition 2.5 A characteristic matrix on $P$ is an integer $(n \times m)$-matrix $\lambda=$ $\left(\lambda_{1}, \ldots, \lambda_{m}\right)$ satisfying the nonsingularity condition for $P$ : if $n$ facets $F_{i_{1}}, \ldots, F_{i_{n}}$ of $P$ meet at a vertex, then $\operatorname{det} \lambda_{\left(i_{1}, \ldots, i_{n}\right)}= \pm 1$, where $\lambda_{\left(i_{1}, \ldots, i_{n}\right)}:=\left(\lambda_{i_{1}}, \ldots, \lambda_{i_{n}}\right)$.

For each quasitoric manifold $M$ over $P$, we obtain the corresponding characteristic matrix $\lambda(M)$ as follows. Let $\pi$ denote the projection from $M$ to $P \cong M / T^{n}$. By definition, we see that every point in $\pi^{-1}\left(\operatorname{rel} \operatorname{int}\left(F_{i}\right)\right)$ has the same isotropy subgroup, which is a one-dimensional subtorus of $T^{n}$. We denote it by $T_{M}\left(F_{i}\right)$. Then, taking primitive vectors $\lambda_{i}={ }^{t}\left(\lambda_{1, i}, \ldots, \lambda_{n, i}\right) \in \mathbb{Z}^{n}(i=1, \ldots, m)$ such that

$$
T_{M}\left(F_{i}\right)=\left\{\left(t^{\lambda_{1, i}}, \ldots, t^{\lambda_{n, i}}\right) \in T^{n}|t \in \mathbb{C},| t \mid=1\right\},
$$

we obtain an integer $(n \times m)$-matrix $\lambda(M):=\left(\lambda_{i, j}\right)$. Clearly, each $\lambda_{i}$ is determined up to sign. Since the $T^{n}$-action on $M$ is locally standard, $\lambda(M)$ satisfies the nonsingularity condition for $P$.

Conversely, we can construct a quasitoric manifold $M(\lambda)$ from a simple polytope $P$ and a characteristic matrix $\lambda$ on $P$. 
Construction 2.6 For each point $q \in P$, we denote the minimal face containing $q$ by $G(q)$. We define the characteristic map $\ell_{\lambda}$ corresponding to $\lambda$, a map from the set of faces of $P$ to the set of subtori of $T^{n}$, as

$$
\ell_{\lambda}\left(F_{i_{1}} \cap \cdots \cap F_{i_{k}}\right):=\operatorname{im}\left(\lambda_{\left(i_{1}, \ldots, i_{k}\right)}: T^{k} \rightarrow T^{n}\right),
$$

where we regard $T$ as $\mathbb{R} / \mathbb{Z}$ and $\lambda_{\left(i_{1}, \ldots, i_{k}\right)}$ as the map induced from the linear map determined by $\lambda_{\left(i_{1}, \ldots, i_{k}\right)}$. Then we obtain a quasitoric manifold $M(\lambda)$ over $P$ by setting

$$
M(\lambda):=\left(T^{n} \times P\right) / \sim_{\lambda},
$$

where $\left(t_{1}, p\right) \sim_{\lambda}\left(t_{2}, q\right)$ if and only if $p=q$ and $t_{1} t_{2}^{-1} \in \ell_{\lambda}(G(q))$. There is a standard differentiable structure given by the atlas $\left\{\pi^{-1}\left(U_{v}\right)\right\}$, where the index $v$ runs over all vertices of $P$ and $U_{v}$ is the open subset obtained by deleting all faces not containing $v$ from $P$. Indeed, each $U_{v}$ is homeomorphic to an open subset of $\mathbb{R}_{+}^{n}$ via an affine map, and hence each $\pi^{-1}\left(U_{v}\right)$ is weakly equivariantly homeomorphic to an open subset of $\mathbb{C}^{n}$. We easily see that the transition maps are diffeomorphic. It is obvious that the $T^{n}$-action on $T^{n} \times P$ by multiplication on the first component descends to a locally standard action on $M(\lambda)$ for which the orbit space is identified with $P$. By definition, $\lambda$ is the characteristic matrix corresponding to $M(\lambda)$.

Definition 2.7 Let us denote the set of all characteristic matrices on a simple polytope $P$ by $\Lambda_{P}$. As in Section $1, \mathcal{M}_{P}$ denotes the set of all weakly equivariant homeomorphism classes of quasitoric manifolds over $P$. Then Construction 2.6 gives the map $\Lambda_{P} \rightarrow \mathcal{M}_{P}, \lambda \mapsto M(\lambda)$. We denote this map by $\phi$.

The following lemma is essential to the classification of quasitoric manifolds up to weakly equivariant homeomorphism; see Davis and Januszkiewicz [5, Lemma 1.4] and Davis [4, page 344].

Lemma 2.8 For any quasitoric manifold $M$ over a simple polytope $P$, there exists a continuous map $s: P \rightarrow M$ such that $\pi \circ s=\mathrm{id}_{P}$.

Then for any quasitoric manifold over $P$, the map $T^{n} \times P \rightarrow M,(t, q) \mapsto t \cdot s(q)$ induces an equivariant homeomorphism from $M(\lambda(M))$ to $M$. We obtain the following proposition.

Proposition 2.9 [5, Proposition 1.8] For any quasitoric manifold over a simple polytope $P, M(\lambda(M))$ is equivariantly homeomorphic to $M$. In particular, the map $\phi: \Lambda_{P} \rightarrow \mathcal{M}_{P}$ is surjective. 
Remark 2.10 Davis and Januszkiewicz [5, Proposition 1.8] state that the correspondence $\lambda \mapsto M(\lambda)$ induces a surjection from $\Lambda_{P}$ to $\widetilde{\mathcal{M}}_{P}$. Here $\widetilde{\mathcal{M}}_{P}$ denotes the set of equivalence classes of quasitoric manifolds $M$ over $P$ equipped with specified projections $\pi: M \rightarrow P$ (namely, equipped with a specified identification $M / T^{n}=P$ ), where an equivalence $f: M \rightarrow N$ is a weakly equivariant homeomorphism such that $\pi=\pi \circ f$.

Next we consider when two characteristic matrices give rise to weakly equivariantly homeomorphic quasitoric manifolds. Let us denote the group of simplicial automorphisms of $K_{P}$ by $\operatorname{Aut}\left(K_{P}\right)$.

Definition 2.11 $\mathrm{GL}(n, \mathbb{Z})$ acts on $\Lambda_{P}$ by left multiplication. Similarly, $(\mathbb{Z} / 2)^{m}$ acts on $\Lambda_{P}$ by multiplication by -1 on each column, and $\operatorname{Aut}\left(K_{P}\right)$ acts by column permutation. We denote $\operatorname{GL}(n, \mathbb{Z}) \backslash \Lambda_{P} /(\mathbb{Z} / 2)^{m}$, the quotient of the biaction, by $\mathcal{X}_{P}$. Then the right $\operatorname{Aut}\left(K_{P}\right)$-action on $\Lambda_{P}$ descends to an action on $\mathcal{X}_{P}$.

Let us show that $\phi: \Lambda_{P} \rightarrow \mathcal{M}_{P}$ descends to $\bar{\phi}: \mathcal{X}_{P} / \operatorname{Aut}\left(K_{P}\right) \rightarrow \mathcal{M}_{P}$. Let $\lambda=$ $\left(\lambda_{1}, \ldots, \lambda_{m}\right), \lambda^{\prime}=\left(\lambda_{1}^{\prime}, \ldots, \lambda_{m}^{\prime}\right)$ be characteristic matrices on a simple polytope $P$, and put $M:=M(\lambda), M^{\prime}:=M\left(\lambda^{\prime}\right)$. Assume there are $\sigma \in \operatorname{Aut}\left(K_{P}\right)$ and $A \in \operatorname{GL}(n, \mathbb{Z})$ such that

$$
\lambda_{\sigma(i)}^{\prime}= \pm A \lambda_{i} \quad(i=1, \ldots, m)
$$

Denote the realization of $\sigma$ by $\bar{f}: P \rightarrow P$ (recall Proposition 2.1) and the automorphism induced from $A$ by $\psi: T^{n} \rightarrow T^{n}$. Then the map

$$
\tilde{f}: T^{n} \times P \rightarrow T^{n} \times P, \quad(t, q) \mapsto(\psi(t), \bar{f}(q))
$$

descends to a homeomorphism $f: M \rightarrow M^{\prime}$ which is weakly equivariant.

Conversely, assume that there is a weakly equivariant homeomorphism $f$ from $M$ to $M^{\prime}$ for an automorphism $\psi$ of $T^{n}$. Let us denote the matrix corresponding to $\psi$ by $A \in \mathrm{GL}(n, \mathbb{Z})$ and the self-homeomorphism of $P$ induced from $f$ (recall Proposition 2.4) by $\bar{f}$. Then we have

$$
\psi\left(T_{M}\left(F_{i}\right)\right)=T_{M^{\prime}}\left(\bar{f}\left(F_{i}\right)\right) \quad(i=1, \ldots, m),
$$

in other words,

$$
\lambda_{\sigma(i)}^{\prime}= \pm A \lambda_{i} \quad(i=1, \ldots, m)
$$

where $\sigma$ is the simplicial automorphism of $K_{P}$ defined by $F_{\sigma(i)}=\bar{f}\left(F_{i}\right)$ for $i=$ $1, \ldots, m$. Hence we see that $\bar{\phi}: \mathcal{X}_{P} / \operatorname{Aut}\left(K_{P}\right) \rightarrow \mathcal{M}_{P}$ is injective. Now we obtain the following proposition. 
Proposition 2.12 For any simple polytope $P, \phi: \Lambda_{P} \rightarrow \mathcal{M}_{P}, \lambda \mapsto M(\lambda)$ descends to a bijection $\bar{\phi}: \mathcal{X}_{P} / \operatorname{Aut}\left(K_{P}\right) \rightarrow \mathcal{M}_{P}$.

Definition 2.13 Let $P$ be a simple polytope. We say that two characteristic matrices on $P$ are equivalent if they are equal in $\mathcal{X}_{P} / \operatorname{Aut}\left(K_{P}\right)$.

\subsection{Moment-angle manifold}

Sometimes it is more useful to regard a quasitoric manifold over $P$ as the orbit space for a free $T^{m-n}$-action on the moment-angle manifold $\mathcal{Z}_{P}$ than to regard it as a product of Construction 2.6; for details, see Buchstaber and Panov [1]. The moment-angle manifold corresponding to a simple $n$-polytope $P$ with $m$ facets is, as a topological space, the subset of $\left(D^{2}\right)^{m}$ defined as

$$
\mathcal{Z}_{P}:=\left\{z=\left(z_{1}, \ldots, z_{m}\right) \in\left(D^{2}\right)^{m} \mid \sigma_{z} \in K_{P}\right\},
$$

where $D^{2}$ denotes the unit closed disk in $\mathbb{C}$ and $\sigma_{z}:=\left\{i|| z_{i} \mid<1\right\}$. For a characteristic matrix $\lambda$ on $P$, let $T_{\lambda}$ be the kernel of the epimorphism from $T^{m}$ to $T^{n}$ induced from $\lambda$. Then $T_{\lambda}$ is an $(m-n)$-dimensional subtorus of $T^{m}$, which acts freely on $\mathcal{Z}_{\boldsymbol{P}}$ by the restriction of the coordinate-wise $T^{m}$-action. The $T^{m}$-action is smooth with respect to the natural differentiable structure on $\mathcal{Z}_{P}$. We can show that the orbit space $\mathcal{Z}_{P} / T_{\lambda}$ is equivariantly homeomorphic to $M(\lambda)$ with respect to the action of $T^{m} / T_{\lambda}$, which is identified with $T^{n}$ through the epimorphism $\lambda: T^{m} \rightarrow T^{n}$.

For example, there is a characteristic matrix $\lambda=(-I \mid 1)$ on $\Delta^{n}$, where $I$ denotes the unit matrix of size $n$ and 1 denotes the column vector with all components equal to one. Since $\mathcal{Z}_{\Delta^{n}}=S^{2 n+1}$ and the one-dimensional torus $T_{\lambda}$ acts diagonally, we see that $M(\lambda)$ is equivariantly homeomorphic to $\mathbb{C P}^{n}$ with the standard $T^{n}$-action.

\subsection{Small covers}

Again let $P$ be an $n$-dimensional simple polytope. Replacing $T^{n}$ by $(\mathbb{Z} / 2)^{n}$ and $\mathbb{C}^{n}$ by $\mathbb{R}^{n}$ in the definition of a quasitoric manifold, we obtain the notion of a small cover over $P$; for details, see Davis and Januszkiewicz [5]. Moreover, replacing $\mathbb{Z}$ by $\mathbb{Z} / 2$, we define a real characteristic matrix on $P$. Then we can construct a small cover $\mathbb{R}_{\mathbb{R}} M(\lambda)$ from a real characteristic matrix $\lambda$, in the same way as in Construction 2.6. We denote the set of all real characteristic matrices on $P$ by $\Lambda_{P}$. Note that $\operatorname{GL}(n, \mathbb{Z} / 2)$ acts on $\mathbb{R}_{P}$ by left multiplication, and $\operatorname{Aut}\left(K_{P}\right)$ acts by column permutation. As in Section 1 , we denote by $\mathbb{R} \mathcal{M}_{P}$ the set of all weakly equivariant homeomorphism classes of small covers over $P$. 
Definition 2.14 Let us denote the map $\mathbb{R}_{P} \rightarrow \mathbb{R}_{P}, \lambda \mapsto \mathbb{R} M(\lambda)$ by $\mathcal{R}_{\mathbb{R}} \phi$, and the quotient $\operatorname{GL}(n, \mathbb{Z} / 2) \backslash_{\mathbb{R}} \Lambda_{P}$ by $\mathbb{R}_{P}$. Then the right $\operatorname{Aut}\left(K_{P}\right)$-action on $\mathbb{R}_{P}$ descends to an action on $\mathbb{R}_{P}$. We say that two real characteristic matrices on $P$ are equivalent if they are equal in $\mathbb{R}_{P} / \operatorname{Aut}\left(K_{P}\right)$.

As for quasitoric manifolds, we get the following proposition for small covers.

Proposition 2.15 For any simple polytope $P, \mathbb{R} \phi: \mathbb{R}_{P} \rightarrow \mathbb{R}_{P}, \lambda \mapsto \mathbb{R} M(\lambda)$ descends to a bijection $\mathbb{R} \bar{\phi}: \mathbb{R}_{P} / \operatorname{Aut}\left(K_{P}\right) \rightarrow \mathbb{R} \mathcal{M}_{P}$.

Replacing $D^{2}$ by the interval $[-1,1]$ and $S^{1}$ by $\{1,-1\}$, we define the real momentangle manifold $\mathbb{R}_{\mathbb{Z}} \mathcal{Z}_{P}$. For a real characteristic matrix $\lambda$ on $P$, let $S_{\lambda}$ be the kernel of the epimorphism from $(\mathbb{Z} / 2)^{m}$ to $(\mathbb{Z} / 2)^{n}$ induced from $\lambda$. In the same way as $\mathcal{Z}_{P}$, we see that the orbit space $\mathbb{Z}_{P} / S_{\lambda}$ is equivariantly homeomorphic to $\mathbb{R} M(\lambda)$ with respect to the action of $(\mathbb{Z} / 2)^{m} / S_{\lambda}$, which is identified with $(\mathbb{Z} / 2)^{n}$ through the epimorphism $\lambda:(\mathbb{Z} / 2)^{m} \rightarrow(\mathbb{Z} / 2)^{n}$. For example, there is a real characteristic matrix $\lambda=(I \mid 1)$ on $\Delta^{n}$. Since $\mathbb{R}_{\mathcal{Z}^{n}}=S^{n}$ and $S_{\lambda}$ acts diagonally, we see that $\mathbb{R} M(\lambda)$ is equivariantly homeomorphic to $\mathbb{R} \mathrm{P}^{n}$ with the standard $(\mathbb{Z} / 2)^{n}$-action.

Then we have the classification of quasitoric manifolds and small covers over a simplex:

Corollary 2.16 For any positive integer $n, \mathcal{M}_{\Delta^{n}}$ (resp. $\left.\mathbb{R}_{\Delta^{n}}\right)$ has only one element, and it is represented by $\mathbb{C P}^{n}$ (resp. $\mathbb{R P}^{n}$ ) with the standard $T^{n}$-action (resp. $(\mathbb{Z} / 2)^{n}$ action).

Let $P$ be a simple $n$-polytope. If $\lambda$ is a characteristic matrix on $P$, then the modulo 2 reduction $\bar{\lambda}$ is a real characteristic matrix on $P$. The involution

$$
T^{n} \times P \rightarrow T^{n} \times P, \quad(t, q) \mapsto\left(t^{-1}, q\right)
$$

descends to an involution on $M(\lambda)$, and we call it the conjugation involution on $M(\lambda)$. Regarding (canonically) $(\mathbb{Z} / 2)^{n}$ as a subset of $T^{n}$, we easily see that the fixed point set for the conjugation involution is $(\mathbb{Z} / 2)^{n}$-stable, and it is equivariantly homeomorphic to $\mathbb{R}_{\mathbb{R}} M(\bar{\lambda})$. Then we can rephrase the lifting problem as follows.

Problem 2.17 For a real characteristic matrix $\lambda$ on a simple polytope $P$, is there a characteristic matrix $\lambda^{\prime}$ on $P$ such that $\lambda$ is the modulo- 2 reduction of $\lambda^{\prime}$ ?

For a real characteristic matrix $\lambda=\left(\lambda_{i, j}\right)$ on $P$, let us define $\tilde{\lambda}=\left(\tilde{\lambda}_{i, j}\right)$ by $\tilde{\lambda}_{i, j} \equiv$ $\lambda_{i, j} \bmod 2$, so that $\tilde{\lambda}_{i, j}=0,1$ for each $i, j$. Since the determinant of any three by three matrix with each component zero or one is between -2 and 2 , we see that, if the dimension of $P$ is not more than three, $\tilde{\lambda}$ is a characteristic matrix on $P$. Then we have the following proposition. 
Proposition 2.18 The answer to the lifting problem is affirmative for any real characteristic matrix on a simple polytope of dimension $\leq 3$.

\subsection{Cohomology}

For the cohomology rings of a quasitoric manifold and a small cover, the following theorems are known; see Davis and Januszkiewicz [5, Theorem 3.1] and [5, Theorem 4.14], and also Buchstaber and Panov [1].

Definition 2.19 Let $P$ be a simple $n$-polytope and $f_{i}$ be the number of $(n-i-1)-$ dimensional faces of $P$. Then the integer vector $\left(f_{0}, \ldots, f_{n-1}\right)$ is called the $f$-vector of $P$. We define the $h$-vector $\left(h_{0}, \ldots, h_{n}\right)$ of $P$ by the equation

$$
h_{0} t^{n}+\cdots+h_{n-1} t+h_{n}=(t-1)^{n}+f_{0}(t-1)^{n-1}+\cdots+f_{n-1}
$$

in the polynomial ring $\mathbb{Z}[t]$.

Note that, conversely, the $h$-vector of a simple polytope determines the $f$-vector.

Theorem 2.20 (Davis and Januszkiewicz) Let $P$ be a simple $n$-polytope and let $\left(h_{0}, \ldots, h_{n}\right)$ be the $h$-vector of $P$.

(i) Suppose that there exists a small cover $M$ over $P$. Let $b_{i}(M)$ be the $i^{\text {th }} \bmod 2$ Betti number of $M$, ie $b_{i}(M):=\operatorname{dim}_{\mathbb{Z} / 2} H_{i}(M ; \mathbb{Z} / 2)$. Then $b_{i}(M)=h_{i}$.

(ii) Suppose that there exists a quasitoric manifold $M$ over $P$. The homology of $M$ vanishes in odd dimensions and is free in even dimensions. Let $b_{2 i}(M)$ be the $2 i^{\text {th }}$ Betti number of $M$. Then $b_{2 i}(M)=h_{i}$.

Let $P$ be a simple polytope with $m$ facets $F_{1}, \ldots, F_{m}$ and $M$ be a quasitoric manifold (resp. small cover) over $P$. We denote the projection from $M$ to $P$ by $\pi$. Then each $\pi^{-1}\left(F_{i}\right)$ is a closed submanifold of dimension $2 n-2$ (resp. $n-1$ ).

Theorem 2.21 (Davis and Januszkiewicz) Let $P$ be an $n$-dimensional simple polytope with $m$ facets and $\lambda=\left(\lambda_{i, j}\right)$ be a characteristic matrix on $P$, and put $M:=M(\lambda)$. Then the integral cohomology ring of $M$ is given by

$$
H^{*}(M ; \mathbb{Z})=\mathbb{Z}\left[v_{1}, \ldots, v_{m}\right] /\left(\mathcal{I}_{P}+\mathcal{J}_{\lambda}\right),
$$

where $v_{i} \in H^{2}(M ; \mathbb{Z})$ is the Poincare dual of the closed submanifold $\pi^{-1}\left(F_{i}\right)$, and $\mathcal{I}_{P}, \mathcal{J}_{\lambda}$ are the ideals

$$
\begin{aligned}
\mathcal{I}_{P} & =\left(v_{i_{1}} \cdots v_{i_{k}} \mid F_{i_{1}} \cap \cdots \cap F_{i_{k}}=\varnothing\right), \\
\mathcal{J}_{\lambda} & =\left(\lambda_{i, 1} v_{1}+\cdots+\lambda_{i, m} v_{m} \mid i=1, \ldots, n\right) .
\end{aligned}
$$


Remark 2.22 Let $\lambda$ and $\lambda^{\prime}$ be two characteristic matrices on $P$. If we assume that $G \lambda=\lambda^{\prime}$, where $G \in \operatorname{GL}(n, \mathbb{Z})$, then $\mathcal{J}_{\lambda}=\mathcal{J}_{\lambda^{\prime}}$. Moreover, if $G \lambda \sigma \epsilon=\lambda^{\prime}$ for $G \in \operatorname{GL}(n, \mathbb{Z}), \sigma \in \operatorname{Aut}\left(K_{P}\right)$ and $\epsilon \in(\mathbb{Z} / 2)^{m}$, then the automorphism of $\mathbb{Z}\left[v_{1}, \ldots, v_{m}\right]$ induced by $\left(v_{1}, \ldots, v_{m}\right) \mapsto\left(v_{1}, \ldots, v_{m}\right) \epsilon \sigma^{-1}$ maps $\mathcal{I}_{P}+\mathcal{J}_{\lambda}$ into $\mathcal{I}_{P}+\mathcal{J}_{\lambda^{\prime}}$.

Theorem 2.23 (Davis and Januszkiewicz) Let $P$ be an $n$-dimensional simple polytope with $m$ facets and $\lambda=\left(\lambda_{i, j}\right)$ be a real characteristic matrix on $P$, and put $M:=\mathbb{R} M(\lambda)$. Then the mod 2 cohomology ring of $M$ is given by

$$
H^{*}(M ; \mathbb{Z} / 2)=(\mathbb{Z} / 2)\left[v_{1}, \ldots, v_{m}\right] /\left(\mathcal{I}_{P}+\mathcal{J}_{\lambda}\right),
$$

where $v_{i} \in H^{1}(M ; \mathbb{Z} / 2)$ is the Poincaré dual of the closed submanifold $\pi^{-1}\left(F_{i}\right)$ and $\mathcal{I}_{P}, \mathcal{J}_{\lambda}$ are the ideals

$$
\begin{aligned}
\mathcal{I}_{P} & =\left(v_{i_{1}} \cdots v_{i_{k}} \mid F_{i_{1}} \cap \cdots \cap F_{i_{k}}=\varnothing\right), \\
\mathcal{J}_{\lambda} & =\left(\lambda_{i, 1} v_{1}+\cdots+\lambda_{i, m} v_{m} \mid i=1, \ldots, n\right) .
\end{aligned}
$$

Moreover, the following theorem is known for the total Stiefel-Whitney class $w(M)$ and the total Pontrjagin class $p(M)$ of a quasitoric manifold or small cover $M$.

Theorem 2.24 (Davis and Januszkiewicz [5, Corollary 6.8]) With the notation in Theorems 2.23 and 2.21, we have the following formulas:

(i) For a small cover $M=\mathbb{R}_{\mathbb{R}} M(\lambda)$,

$$
w(M)=\prod_{i=1}^{m}\left(1+v_{i}\right) \quad \text { and } \quad p(M)=1 .
$$

(ii) For a quasitoric manifold $M=M(\lambda)$,

$$
w(M)=\prod_{i=1}^{m}\left(1+v_{i}\right) \quad \text { and } \quad p(M)=\prod_{i=1}^{m}\left(1-v_{i}^{2}\right) .
$$

For convenience, in subsequent arguments we denote the integral cohomology ring of $M(\lambda)$ by $H^{*}(\lambda)$ for a characteristic matrix $\lambda$. Similarly, for a real characteristic matrix $\lambda^{\prime}$, we denote the mod 2 cohomology ring of $\mathbb{R} M\left(\lambda^{\prime}\right)$ by $H^{*}\left(\lambda^{\prime}\right)$.

\section{Cyclic polytopes and connected sums}

First we review the definition of a cyclic polytope and its combinatorial structure. See Ziegler [8] for details. 


\subsection{Cyclic polytopes}

Recall that a convex polytope is called simplicial if all facets are simplices. By definition, the dual of a simplicial polytope is simple and vice versa. For a combinatorial polytope $P$, we denote the dual of $P$ by $P^{*}$.

Given an increasing sequence $t_{1}<\cdots<t_{m}$ of real numbers, let $C^{n}\left(t_{1}, \ldots, t_{m}\right)$ be the convex hull of $m$ points

$$
T_{1}:=\left(t_{1}, t_{1}^{2}, \ldots, t_{1}^{n}\right), \quad \ldots, \quad T_{m}:=\left(t_{m}, t_{m}^{2}, \ldots, t_{m}^{n}\right)
$$

in $\mathbb{R}^{n}$ for $n<m$. Then $C^{n}\left(t_{1}, \ldots, t_{m}\right)$ is an $n$-dimensional simplicial polytope with $m$ vertices $T_{1}, \ldots, T_{m}$. The combinatorial structure of $C^{n}\left(t_{1}, \ldots, t_{m}\right)$ is characterized by the following result.

Theorem 3.1 (Gale's evenness condition, [8, Theorem 0.7]) Let $T$ denote the vertex set $\left\{T_{1}, \ldots, T_{m}\right\}$. Then an $n$-subset $S \subseteq T$ forms a facet of $C^{n}\left(t_{1}, \ldots, t_{m}\right)$ if and only if any two elements in $T \backslash S$ are separated by an even number of elements from $S$ in the sequence $\left(T_{1}, \ldots, T_{m}\right)$.

It follows immediately from this theorem that the combinatorial type of $C^{n}\left(t_{1}, \ldots, t_{m}\right)$ depends only on $m$ and $n$. Then we denote this combinatorial polytope by $C^{n}(m)$ and call it the cyclic $n$-polytope with $m$ vertices. In this paper, we are particularly interested in the dual simple polytope $C^{n}(m)^{*}$. For convenience, we restate Gale's evenness condition in terms of $C^{n}(m)^{*}$.

Theorem 3.2 (dual evenness condition) There is an order of the facets of $C^{n}(m)^{*}$, say $F_{1}, \ldots, F_{m}$, such that $F_{i_{1}}, \ldots, F_{i_{n}}$ meet at a vertex if and only if $\left\{i_{1}, \ldots, i_{n}\right\}$ is the disjoint union of some $I_{j}$ for $j$ in $\{0, \ldots, m\}$, where $I_{j}:=\{j, j+1\} \cap\{1, \ldots, m\}$.

\subsection{Connected sums}

Next we introduce the connected sum of characteristic (or real characteristic) matrices. Let us first recall the connected sum of two combinatorial simple polytopes. For the formal definition, see Buchstaber and Panov [1] and Ziegler [8, Section 2.6]. Let $P$ and $Q$ be $n$-dimensional combinatorial simple polytopes with facets $F_{1}, \ldots, F_{m_{1}}$ and $G_{1}, \ldots, G_{m_{2}}$ respectively, and put $F:=\left\{F_{1}, \ldots, F_{m_{1}}\right\}$ and $G:=\left\{G_{1}, \ldots, G_{m_{2}}\right\}$. Take two vertices $v \in P$ and $w \in Q$. Denote the sets of the facets meeting at $v$ and $w$ by $F_{v}=\left\{F_{i_{1}}, \ldots, F_{i_{n}}\right\}$ and $G_{w}=\left\{G_{j_{1}}, \ldots, G_{j_{n}}\right\}$ respectively, and fix a one-to-one correspondence $\tau:\left\{i_{1}, \ldots, i_{n}\right\} \rightarrow\left\{j_{1}, \ldots, j_{n}\right\}$. "Cutting off" the vertices $v$ and $w$ from $P$ and $Q$ respectively, we obtain simple polytopes $P^{\prime}$ and $Q^{\prime}$, each of which has 
a new simplex facet. Then, after a projective transformation, we can "glue" $P^{\prime}$ to $Q^{\prime}$ along the simplex facets, using $\tau$. Thus we obtain the connected sum $P \#_{\tau} Q$, a simple polytope the set of whose facets can be identified with $F \cup_{\tau} G$, the quotient of the equivalence relation $\sim_{\tau}$ on $F \amalg G$ defined by $F_{i_{k}} \sim_{\tau} G_{\tau\left(i_{k}\right)}(k=1, \ldots, n)$. What is important is that $K_{P \#_{\tau} Q}$ is identified with $K_{P} \cup_{\tau} K_{Q} \backslash\{\sigma\}$ as an abstract simplicial complex on $F \cup_{\tau} G$, where $\sigma$ denotes the simplex coming from $\left\{i_{1}, \ldots, i_{n}\right\} \in K_{P}$.

Moreover, suppose that there exist (real) characteristic matrices $\lambda=\left(\lambda_{1}, \ldots, \lambda_{m_{1}}\right)$ and $\lambda^{\prime}=\left(\lambda^{\prime}{ }_{1}, \ldots, \lambda^{\prime}{ }_{m_{2}}\right)$ on $P$ and $Q$ respectively. If they satisfy the condition $\lambda_{i_{k}}=$ $\lambda^{\prime}{ }_{\tau\left(i_{k}\right)}$ for $k=1, \ldots, n$, we obtain the connected sum $\lambda \#_{\tau} \lambda^{\prime}$, a (real) characteristic matrix on $P \#_{\tau} Q$, as follows: Number the facets of $P \#_{\tau} Q$ from 1 to $m:=m_{1}+m_{2}-n$, and define $\lambda \#_{\tau} \lambda^{\prime}=\left(\lambda^{\prime \prime}{ }_{1}, \ldots, \lambda^{\prime \prime}{ }_{m}\right)$ by $\lambda^{\prime \prime}{ }_{k}:=\lambda_{i}$ if the $k^{\text {th }}$ facet of $\lambda \#_{\tau} \lambda^{\prime}$ corresponds to $F_{i}$, and $\lambda^{\prime \prime}{ }_{k}:=\lambda^{\prime}{ }_{j}$ if the $k^{\text {th }}$ facet of $\lambda \#_{\tau} \lambda^{\prime}$ corresponds to $G_{j}$. It is easy to observe that $M\left(\lambda \#_{\tau} \lambda^{\prime}\right)$ (resp. $\mathbb{R} M\left(\lambda \#_{\tau} \lambda^{\prime}\right)$ ) is homeomorphic to $M(\lambda) \# M\left(\lambda^{\prime}\right)$ (resp. $\mathbb{R} M(\lambda) \#_{\mathbb{R}} M\left(\lambda^{\prime}\right)$ ) with respect to the proper orientations of $M(\lambda)$ and $M\left(\lambda^{\prime}\right)$.

Conversely, suppose that a simple $n$-polytope $P$ is decomposed into the connected sum of simple polytopes $P^{\prime}, P^{\prime \prime}$. Denote the facets of $P$ by $F_{1}, \ldots, F_{m}$. For simplicity, we assume that the facets $F_{1}, \ldots, F_{k}$ come from $P^{\prime}$ and $F_{k-n+1}, \ldots, F_{m}$ from $P^{\prime \prime}$. Let $\lambda=\left(\lambda_{1}, \ldots, \lambda_{m}\right)$ be a (real) characteristic matrix. If $\lambda$ satisfies the condition $\operatorname{det}\left(\lambda_{k-n+1}, \ldots, \lambda_{k}\right)= \pm 1$, then $\lambda=\lambda^{\prime} \#_{\tau} \lambda^{\prime \prime}$, where $\lambda^{\prime}=\left(\lambda_{1}, \ldots, \lambda_{k}\right)$ and $\lambda^{\prime \prime}=\left(\lambda_{k-n+1}, \ldots, \lambda_{m}\right)$ are (real) characteristic matrices on $P^{\prime}$ and $P^{\prime \prime}$ respectively, and $\tau:\{k-n+1, \ldots, k\} \rightarrow\{1, \ldots, n\}$ maps $i$ to $i+n-k$. Thus we have the following lemma.

Lemma 3.3 Let $P$ be a simple $n$-polytope with $m$ facets $F_{1}, \ldots, F_{m}$ and $\lambda$ be a (real) characteristic matrix on $P$. Suppose that $P$ is decomposed into the connected sum of two simple polytopes $P^{\prime}, P^{\prime \prime}$, and the $n$ facets $F_{i_{1}}, \ldots, F_{i_{n}}$ come from both $P^{\prime}$ and $P^{\prime \prime}$. If the condition $\operatorname{det} \lambda_{\left(i_{1}, \ldots, i_{n}\right)}= \pm 1$ holds, then $\lambda$ is decomposed into the connected sum of (real) characteristic matrices on $P^{\prime}$ and $P^{\prime \prime}$. In particular, $M(\lambda)$ (resp. $\mathbb{R} M(\lambda)$ ) is decomposed into the connected sum of quasitoric manifolds (resp. small covers) over $P^{\prime}$ and $P^{\prime \prime}$.

\subsection{Conventions and remarks}

In subsequent sections, we adopt the following conventions:

(i) If $m-n>2$, we always label the facets of $C^{n}(m)^{*}$ by $F_{1}, \ldots, F_{m}$ so that the dual evenness condition (Theorem 3.2) works.

(ii) For an $(n \times m)$-matrix $\lambda$, we denote the minor $\operatorname{det} \lambda_{\left(i_{1}, \ldots, i_{n}\right)}$ by $\left|i_{1}, \ldots, i_{n}\right|_{\lambda}$, or simply $\left|i_{1}, \ldots, i_{n}\right|$ if $\lambda$ is obvious. 
(iii) For a simple polytope $P$, we identify $\mathcal{M}_{P}\left(\right.$ resp. $\left.\mathbb{R}_{\mathbb{P}} \mathcal{M}_{P}\right)$ with $\mathcal{X}_{P} / \operatorname{Aut}\left(K_{P}\right)$ (resp. $\left.\mathbb{R}_{P} / \operatorname{Aut}\left(K_{P}\right)\right)$. Then, for an element $[M]$ of $\mathcal{M}_{P}\left(\right.$ resp. $\left.\mathbb{R} \mathcal{M}_{P}\right)$, we say that a (real) characteristic matrix $\lambda$ is a representative of $[M]$ if $M(\lambda)$ (resp. $\mathbb{R} M(\lambda)$ ) is a representative of $[M]$.

Note that since $F_{1}, \ldots, F_{n}$ meet at a vertex in $C^{n}(m)^{*}$, we can take a representative

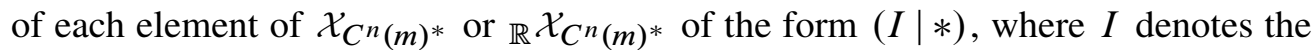
unit matrix of size $n$ and $*$ denotes some $(n \times(m-n))$-matrix. We will generally take such a representative.

For a characteristic matrix $\lambda=(I \mid *)$ on $C^{n}(m)^{*}$, the ideal $\mathcal{J}_{\lambda}$ in Theorem 2.21 is reduced to

$$
\mathcal{J}_{\lambda}=\left(v_{i}+\lambda_{i, n+1} v_{n+1}+\cdots+\lambda_{i, m} v_{m} \mid i=1, \ldots, n\right) .
$$

Recall that a missing face of an abstract simplicial complex $K$ on $\{1, \ldots, m\}$ is a minimal subset of $\{1, \ldots, m\}$ which does not belong to $K$. Let us denote the set of all missing faces of $K$ by $\operatorname{Miss}(K)$. Then for any simple polytope $P$, the ideal $\mathcal{I}_{P}$ in Theorem 2.21 is reduced to

$$
\mathcal{I}_{P}=\left(v_{i_{1}} \cdots v_{i_{k}} \mid\left\{i_{1}, \ldots, i_{k}\right\} \in \operatorname{Miss}\left(K_{P}\right)\right) .
$$

Thus we can restate the theorem as follows.

Theorem 3.4 Let $\lambda=\left(\lambda_{i, j}\right)=(I \mid *)$ be a characteristic matrix on $C^{n}(m)^{*}$. Set $v_{i}^{\prime}:=\lambda_{i, n+1} v_{n+1}+\cdots+\lambda_{i, m} v_{m} \in \mathbb{Z}\left[v_{n+1}, \ldots, v_{m}\right]$ for $i=1, \ldots, n$, and $v_{i}^{\prime}:=v_{i}$ for $i=n+1, \ldots, m$. Then the cohomology ring $H^{*}(\lambda)=H^{*}(M ; \mathbb{Z})$ is given by

$$
H^{*}(\lambda)=\mathbb{Z}\left[v_{n+1}, \ldots, v_{m}\right] / \mathcal{I}_{\lambda},
$$

where each $v_{i}$ has degree two and $\mathcal{I}_{\lambda}$ is the ideal

$$
\mathcal{I}_{\lambda}=\left(v_{i_{1}}^{\prime} \cdots v_{i_{k}}^{\prime} \mid\left\{i_{1}, \ldots, i_{k}\right\} \in \operatorname{Miss}\left(K_{P}\right)\right) .
$$

We use this expression for the cohomology ring in subsequent sections.

\section{Classification for $C^{2}(m)^{*}$}

$C^{2}(m)^{*}$ is obviously equal to the convex $m$-gon. As an application of the connected sum operation, we consider the topological classification of quasitoric manifolds and small covers over a convex polygon. This classification has already appeared in Davis and Januszkiewicz [5], and for small covers, the proof below goes along the same lines. Let us suppose that the facets $F_{1}, \ldots, F_{m}$ of the convex $m$-gon are numbered clockwise. We will make use of the following easy lemmas. 
Lemma 4.1 Let $\lambda=\left(\lambda_{1}, \ldots, \lambda_{m}\right)$ be a (real) characteristic matrix on the convex $m$-gon. Suppose that $i, j \in\{1, \ldots, m\}(i<j)$ satisfy $|i, j|_{\lambda}= \pm 1$ and $F_{i} \cap F_{j}=\varnothing$. Then, setting $d:=j-i+1, \lambda^{\prime}:=\left(\lambda_{i}, \ldots, \lambda_{j}\right)$ and $\lambda^{\prime \prime}=\left(\lambda_{j}, \ldots, \lambda_{m}, \lambda_{1}, \ldots, \lambda_{i}\right)$ are (real) characteristic matrices on the convex $d$-gon and the convex $(m-d+2)-$ gon respectively. Moreover, $\lambda$ decomposes into the connected sum of $\lambda^{\prime}$ and $\lambda^{\prime \prime}$.

Proof It follows immediately from Lemma 3.3.

Lemma 4.2 For any integer matrix $\left(\begin{array}{ll}a & c \\ b & d\end{array}\right)$ of determinant \pm 1 , if $|a|<|b|$, then we have $|c| \leq|d|$.

First, we consider the classification of small covers.

Theorem 4.3 If $m>4$, any small cover over a convex polygon is homeomorphic to the connected sum of copies of $S^{1} \times S^{1}$ and $\mathbb{R P}^{2}$.

Proof Let $\lambda$ be a real characteristic matrix on the convex $m$-gon, where $m>4$. First we show that $\lambda$ is decomposed into the connected sum of real characteristic matrices on two convex polygons. By the nonsingularity condition, we have $|2,3|=|1, m|=1$. Then we can assume that $\lambda$ is of the form

$$
\lambda=\left(\begin{array}{ll|lll}
1 & 0 & 1 & \cdots & b \\
0 & 1 & a & \cdots & 1
\end{array}\right) .
$$

If $a=1$, we have $|1,3|=1$. If $a=0$, we have $|3, m|=1$. Then we see that $\lambda$ is decomposed into a connected sum by Lemma 4.1.

Denote the convex tetragon by $P_{4}$. By a direct calculation, we see that there exist exactly two elements in $\mathbb{R}_{P_{4}}$, which are represented by

$$
\left(\begin{array}{ll|ll}
1 & 0 & 1 & 0 \\
0 & 1 & 0 & 1
\end{array}\right), \quad\left(\begin{array}{ll|ll}
1 & 0 & 1 & 1 \\
0 & 1 & 0 & 1
\end{array}\right)
$$

The left matrix corresponds to the small cover $S^{1} \times S^{1}$, and the right one is decomposed into a connected sum. Then we obtain the theorem by induction on $m$.

By the classification theorem for closed surfaces, we obtain the topological classification of small covers over a convex polygon as follows.

Corollary 4.4 A small cover over a convex polygon is homeomorphic to the connected sum of copies of $S^{1} \times S^{1}$ or that of $\mathbb{R P}^{2}$. 
Next, we consider quasitoric manifolds over a convex polygon. We will show their topological classification without using the results of Orlik and Raymond [7].

Lemma 4.5 If $m>4$, any characteristic matrix on the convex $m$-gon is decomposed into the connected sum of characteristic matrices on two convex polygons.

Proof As in the proof of Theorem 4.3, we can assume that a characteristic matrix on the convex $m$-gon has the form

$$
\lambda=\left(\begin{array}{ll|lllll}
1 & 0 & 1 & c_{1} & \cdots & c_{m-4} & b \\
0 & 1 & a & d_{1} & \cdots & d_{m-4} & 1
\end{array}\right) .
$$

If $a=0, \pm 1$ or $b=0, \pm 1$, we obtain the assertion by Lemma 4.1, so we assume $|a|>1$ and $|b|>1$. Then, by Lemma 4.2, we have $\left|c_{i}\right|=\left|d_{i}\right|$ for some $i$. Since each column vector is primitive, we have $c_{i}, d_{i}= \pm 1$ and therefore $|1, i|= \pm 1$. Then the proof is completed by Lemma 4.1 .

By a direct calculation, we see that $\mathcal{M}_{P_{4}}$ consists of the classes represented by

$$
\lambda_{k}=\left(\begin{array}{ll|ll}
1 & 0 & 1 & k \\
0 & 1 & 0 & 1
\end{array}\right), \quad \lambda^{\prime}=\left(\begin{array}{ll|ll}
1 & 0 & 1 & 2 \\
0 & 1 & 1 & 1
\end{array}\right)
$$

where $k$ is an integer. We can see that $M\left(\lambda_{k}\right)$ is weakly equivariantly homeomorphic to the Hirzebruch surface $H_{k}$, and $M\left(\lambda^{\prime}\right)$ is homeomorphic to $\mathbb{C P}^{2} \# \mathbb{C P}{ }^{2}$. Recall that the Hirzebruch surface $H_{k}$ is homeomorphic to $S^{2} \times S^{2}$ if $k$ is even, and homeomorphic to $\mathbb{C P}^{2} \# \overline{\mathbb{C P}^{2}}$ if $k$ is odd. Then we have the following theorem.

Theorem 4.6 A quasitoric manifold over a convex polygon is homeomorphic to a connected sum of copies of $\mathbb{C P}^{2}, \overline{\mathbb{C P}^{2}}$ and $S^{2} \times S^{2}$.

As is well-known, $\mathbb{C P} \#\left(S^{2} \times S^{2}\right)$ is homeomorphic to $\mathbb{C P}^{2} \# \mathbb{C P} \mathbb{P}^{2} \overline{\mathbb{C P}}$. (This can also be shown using characteristic matrices.) Then we obtain the classification below.

Corollary 4.7 A quasitoric manifold over a convex polygon is homeomorphic to a connected sum of copies of $S^{2} \times S^{2}$ or a connected sum of copies of $\mathbb{C P}^{2}$ and $\overline{\mathbb{C P}^{2}}$.

Remark 4.8 Comparing their signatures, we obtain the cohomological rigidity of the class consisting of the connected sums in Corollary 4.7. Namely, the class of quasitoric manifolds over a convex polygon is cohomologically rigid. 


\section{Classification for $C^{3}(m)^{*}$}

First, we list all maximal faces of $K_{C^{3}(m)^{*}}$ :

$$
\begin{array}{ll}
\{1, i, i+1\} & (i=2, \ldots, m-1) \\
\{i, i+1, m\} & (i=1, \ldots, m-2)
\end{array}
$$

Let $F_{1}, \ldots, F_{m_{1}}$ be the facets of $C^{3}\left(m_{1}\right)^{*}$ and $F_{1}^{\prime}, \ldots, F_{m_{2}}^{\prime}$ those of $C^{3}\left(m_{2}\right)^{*}$. Take vertices $v=F_{1} \cap F_{m_{1}-1} \cap F_{m_{1}}, w=F_{1}^{\prime} \cap F_{2}^{\prime} \cap F_{m_{2}}^{\prime}$, and put $m:=m_{1}+m_{2}-3$. The connected sum $C^{3}\left(m_{1}\right)^{*} \#_{\rho} C^{3}\left(m_{2}\right)^{*}$ is equal to $C^{3}(m)^{*}$, where

$$
\rho:\left\{1, m_{1}-1, m_{1}\right\} \rightarrow\left\{1,2, m_{2}\right\}, \quad 1 \mapsto 1, m_{1}-1 \mapsto 2, m_{1} \mapsto m_{2} .
$$

We label the facets of $C^{3}\left(m_{1}\right)^{*} \#_{\rho} C^{3}\left(m_{2}\right)^{*}$ by $F_{1}^{\prime \prime}, \ldots, F_{m}^{\prime \prime}$ as

$$
\begin{aligned}
& F_{1}^{\prime \prime}=F_{1}=F_{1}^{\prime}, \quad F_{2}^{\prime \prime}=F_{2}, \quad \ldots \quad F_{m_{1}-2}^{\prime \prime}=F_{m_{1}-2}, \\
& F_{m_{1}-1}^{\prime \prime}=F_{m_{1}-1}=F_{2}^{\prime}, \quad F_{m_{1}}^{\prime \prime}=F_{3}^{\prime}, \quad \ldots \quad F_{m-1}^{\prime \prime}=F_{m_{2}-1}^{\prime}, \quad F_{m}^{\prime \prime}=F_{m_{1}}=F_{m_{2}}^{\prime} \text {. }
\end{aligned}
$$

Then the dual evenness condition works for $C^{3}\left(m_{1}\right)^{*} \#_{\rho} C^{3}\left(m_{2}\right)^{*}=C^{3}(m)^{*}$ with this labeling. We have the following lemma.
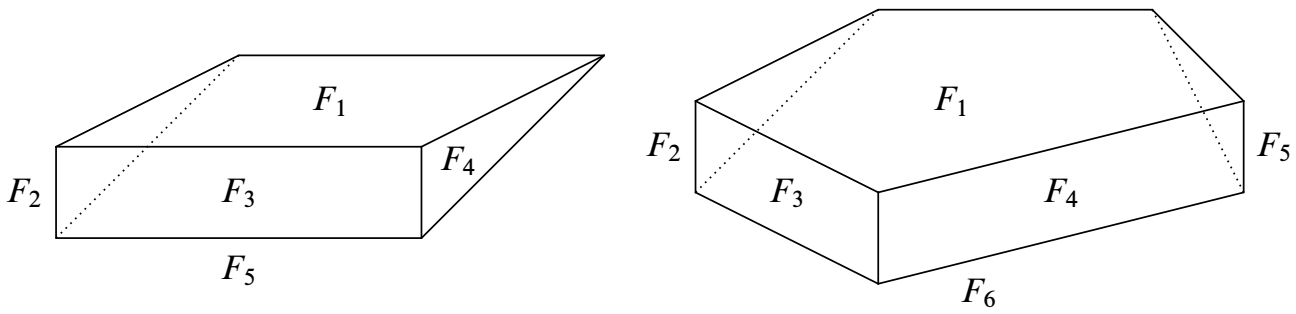

Figure 1: The shapes of $C^{3}(5)^{*}$ and $C^{3}(6)^{*}$

Lemma 5.1 Let $\lambda$ be a (real) characteristic matrix on $C^{3}(m)^{*}$. If the condition $|1, k, m|_{\lambda}= \pm 1$ holds for some $2<k<m-1$, then $\lambda$ is decomposed into the connected sum of two characteristic matrices on $C^{3}(k+1)^{*}$ and $C^{3}(m-k+2)^{*}$.

Proof This follows immediately from Lemma 3.3 and the above discussion.

Definition 5.2 A (real) characteristic matrix on $C^{3}(m)^{*}$ is said to be decomposable if the condition $|1, k, m|_{\lambda}= \pm 1$ holds for some $2<k<m-1$; otherwise it is said to be indecomposable. Moreover, we say that a quasitoric manifold over $C^{3}(m)^{*}$ is decomposable (resp. indecomposable) if the corresponding (real) characteristic matrix is decomposable (resp. indecomposable). 
Lemma 5.3 If $m>5, \operatorname{Aut}\left(K_{C^{3}(m)^{*}}\right)$ is generated by $\sigma=\left(\begin{array}{ccccc}1 & 2 & \cdots & m-1 & m \\ m & m-1 & \cdots & 2 & 1\end{array}\right)$ and $\tau=\left(\begin{array}{ll}1 & m\end{array}\right)$, and if $m=5$, it is generated by $\left(\begin{array}{lllll}1 & 2 & 3 & 4 & 5 \\ 3 & 4 & 5 & 2 & 1\end{array}\right)$ and $\left(\begin{array}{ll}1 & 5\end{array}\right)$.

Proof First, we can directly check that these permutations certainly give automorphisms of $K_{C^{3}(m)^{*}}$. Considering the number of vertices of each facets, we see that there exists no other permutation of $\{1, \ldots, 5\}$ which can be an automorphism of $K_{C^{3}(5)^{*}}$. Similarly, for any $\rho \in \operatorname{Aut}\left(K_{C^{3}(m)^{*}}\right)$ for $m>5$, we have $\{\rho(1), \rho(m)\}=\{1, m\}$ and $\{\rho(2), \rho(m-1)\}=\{2, m-1\}$. Composing $\rho$ with $\sigma$ and $\tau$ if necessary, we can assume that $\rho(1)=1, \rho(2)=2, \rho(m-1)=m-1, \rho(m)=m$. Then we have $\rho(3)=3$ since $\rho(\{1,2,3\})=\{1,2, \rho(3)\} \in K_{C^{3}(m)^{*}}$ and $\rho(3) \neq m$. In the same way, we have $\rho(4)=4, \ldots, \rho(m-2)=m-2$.

Let us remark that the action of $\operatorname{Aut}\left(K_{\left.C^{3}(m)^{*}\right)}\right)$ preserves decomposability/indecomposability of (real) characteristic matrices. Then we say that an element $\mathcal{M}$ of $\mathcal{M}_{C^{3}(m)}{ }^{*}$ (resp. $\mathbb{R}_{C^{3}(m)^{*}}$ ) is decomposable/indecomposable if a representative (real) characteristic matrix of $\mathcal{M}$ is decomposable/indecomposable.

To classify quasitoric manifolds over $C^{3}(6)^{*}$ topologically, we start by listing all indecomposable characteristic matrices on $C^{3}(6)^{*}$ up to equivalence. Then we will show that the class of indecomposable quasitoric manifolds over $C^{3}(6)^{*}$ is cohomologically rigid, ie their homeomorphism classes are distinguished by their cohomology rings. In the statement of Proposition 5.4, by a matrix $*$ we mean the whole characteristic matrix $(I \mid *)$.

Proposition 5.4 All indecomposable elements of $\mathcal{X}_{C^{3}(6) *}$ are represented by the characteristic matrices

$$
\begin{aligned}
& \lambda_{1}=\left(\begin{array}{lll}
0 & 0 & 1 \\
1 & 1 & 2 \\
2 & 1 & 1
\end{array}\right), \lambda_{1}^{\prime}=\left(\begin{array}{lll}
0 & 0 & 1 \\
1 & 2 & 3 \\
1 & 1 & 1
\end{array}\right), \\
& \lambda_{2}=\left(\begin{array}{lll}
1 & 1 & 1 \\
1 & 1 & 2 \\
2 & 1 & 1
\end{array}\right), \lambda_{2}^{\prime}=\left(\begin{array}{rrr}
-1 & -1 & 1 \\
1 & 1 & -2 \\
1 & 0 & 1
\end{array}\right), \lambda_{2}^{\prime \prime}=\left(\begin{array}{lll}
0 & 1 & 1 \\
1 & 1 & 3 \\
1 & 0 & 1
\end{array}\right), \lambda_{2}^{\prime \prime \prime}=\left(\begin{array}{lll}
0 & 1 & 1 \\
1 & 2 & 3 \\
1 & 1 & 1
\end{array}\right), \\
& \lambda_{d}=\left(\begin{array}{lll}
0 & 0 & 1 \\
1 & 1 & d \\
1 & 0 & 1
\end{array}\right),
\end{aligned}
$$

where $d \leq-2$ or $d \geq 3$, and the actions of $\sigma, \tau \in$ Aut $\left(K_{C^{3}(6)^{*}}\right)$ are illustrated as follows: 


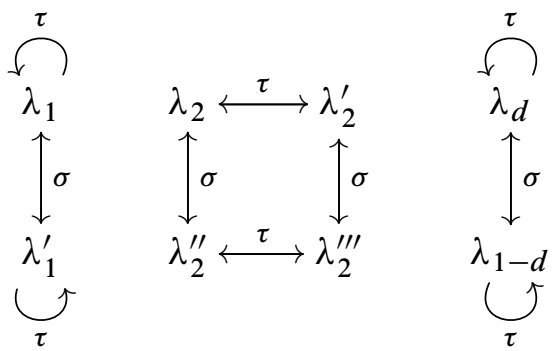

Proof Let $\lambda$ be an indecomposable characteristic matrix on $C^{3}(6)^{*}$. As $|1,3,4|= \pm 1$, $|1,2,6|= \pm 1$ and $|2,3,6|= \pm 1$, we can assume that $\lambda$ has the form

$$
\lambda=\left(\begin{array}{cccccc}
1 & 0 & 0 & a_{1} & b_{1} & 1 \\
0 & 1 & 0 & 1 & b_{2} & c_{2} \\
0 & 0 & 1 & a_{3} & b_{3} & 1
\end{array}\right) .
$$

Moreover, $a_{3} \neq 0$ from the indecomposability, and then we can assume $a_{3}>0$ by multiplication by -1 on the second and fourth columns and the middle row, if necessary. Similarly, from the indecomposability, we have $c_{2} \neq 0, \pm 1$. If $a_{3}=1$, we have $c_{2} \geq 3$ or $c_{2} \leq-2$, once more from the indecomposability. If $a_{3} \geq 2$, we obtain $b_{2}, b_{3}= \pm 1$ by Lemma 4.2, and hence we have $a_{3}=2$. By multiplication with -1 on the fifth column if necessary, we can assume $b_{2}=b_{3}=1$.

To summarize, we only have to consider two cases, (i) $a_{3}=1$ and $c_{2} \geq 3$ or $c_{2} \leq-2$ and (ii) $a_{3}=c_{2}=2$ and $b_{2}=b_{3}=1$. By a direct calculation, in case (i) we obtain the characteristic matrices $\lambda_{1}^{\prime}, \lambda_{2}^{\prime}, \lambda_{2}^{\prime \prime}, \lambda_{2}^{\prime \prime \prime}$ and $\lambda_{d}$ for $d \leq-2$ or $d \geq 3$ in the statement. Similarly, in case (ii) we obtain $\lambda_{1}$ and $\lambda_{2}$.

Then we take $M_{1}:=M\left(\lambda_{1}^{\prime}\right), M_{2}:=M\left(\lambda_{2}^{\prime \prime \prime}\right)$ and $M_{d}:=M\left(\lambda_{d}\right)$ for $d \geq 3$, and also $A_{1}:=H^{*}\left(\lambda_{1}^{\prime}\right), A_{2}:=H^{*}\left(\lambda_{2}^{\prime \prime \prime}\right)$ and $A_{d}:=H^{*}\left(\lambda_{d}\right)$ for $d \geq 3$. Notice that $\operatorname{Miss}\left(K_{C^{3}(6)^{*}}\right)$ consists of $\{2,4\},\{2,5\},\{3,5\},\{1,3,6\}$ and $\{1,4,6\}$, so $\mathcal{I}_{C^{3}(6)^{*}}$ in Theorem 2.21 is generated by $v_{2} v_{4}, v_{2} v_{5}, v_{3} v_{5}, v_{1} v_{3} v_{6}$ and $v_{1} v_{4} v_{6}$. Then, putting $X:=v_{4}, Y:=v_{5}, Z:=v_{6}$ in Theorem 3.4, we have

$$
A_{i}=\mathbb{Z}[X, Y, Z] /\left(\mathcal{I}_{i}^{4}+\mathcal{I}_{i}^{6}\right),
$$

where $\mathcal{I}_{i}^{4}$ and $\mathcal{I}_{i}^{6}$ are the ideals

$$
\begin{aligned}
\mathcal{I}_{1}^{4}=\mathcal{I}_{2}^{4} & =(X(X+2 Y+3 Z), Y(X+2 Y+3 Z), Y(X+Y+Z)), \\
\mathcal{I}_{1}^{6} & =\left(Z^{2}(X+Y+Z), Z^{2} X\right), \\
\mathcal{I}_{2}^{6} & =(Z(Y+Z)(X+Y+Z), Z X(Y+Z)), \\
\mathcal{I}_{d}^{4} & =(X(X+Y+d Z), Y(X+Y+d Z), Y(X+Z)),
\end{aligned}
$$




$$
\mathcal{I}_{d}^{6}=\left(Z^{2}(X+Z), Z^{2} X\right) .
$$

Suppose that there exists a graded ring isomorphism from $A_{i}$ to $A_{j}$, and denote it by $\phi$. We may regard $\phi$ as a graded ring automorphism of $\mathbb{Z}[X, Y, Z]$ such that $\phi\left(\left(\mathcal{I}_{i}^{4}+\mathcal{I}_{i}^{6}\right)\right) \subseteq\left(\mathcal{I}_{j}^{4}+\mathcal{I}_{j}^{6}\right)$. We put $\phi(X)=a_{1} X+b_{1} Y+c_{1} Z, \phi(Y)=$ $a_{2} X+b_{2} Y+c_{2} Z$ and $\phi(Z)=a_{3} X+b_{3} Y+c_{3} Z$, and write $a=\left(a_{1}, a_{2}, a_{3}\right)$, $b=\left(b_{1}, b_{2}, b_{3}\right)$ and $c=\left(c_{1}, c_{2}, c_{3}\right)$. Thus we may denote $\phi$ by the matrix $\left({ }^{t} a,{ }^{t} b,{ }^{t} c\right)$.

Lemma 5.5 If $i=1,2$ and $j=d \geq 3$, then $c \equiv \pm(1,1,1) \bmod 3$.

Proof Since $Z^{2}$ does not appear in $\mathcal{I}_{d}^{4}$, the coefficients of $Z^{2}$ in $\phi(X(X+2 Y+3 Z))$, $\phi(Y(X+2 Y+3 Z))$ and $\phi(Y(X+Y+Z))$ are zero. Hence we obtain

$$
\left(\begin{array}{c}
c_{1}\left(c_{1}+2 c_{2}+3 c_{3}\right) \\
c_{2}\left(c_{1}+2 c_{2}+3 c_{3}\right) \\
c_{2}\left(c_{1}+c_{2}+c_{3}\right)
\end{array}\right)=0
$$

Similarly, comparing the coefficients of $X^{2}$ and those of $Z X$ in $\phi(X(X+2 Y+3 Z))$, $\phi(Y(X+2 Y+3 Z))$ and $\phi(Y(X+Y+Z))$, we obtain

$$
d\left(\begin{array}{c}
c_{1}\left(a_{1}+2 a_{2}+3 a_{3}\right)+a_{1}\left(c_{1}+2 c_{2}+3 c_{3}\right) \\
c_{2}\left(a_{1}+2 a_{2}+3 a_{3}\right)+a_{2}\left(c_{1}+2 c_{2}+3 c_{3}\right) \\
c_{2}\left(a_{1}+a_{2}+a_{3}\right)+a_{2}\left(c_{1}+c_{2}+c_{3}\right)
\end{array}\right)=\left(\begin{array}{c}
a_{1}\left(a_{1}+2 a_{2}+3 a_{3}\right) \\
a_{2}\left(a_{1}+2 a_{2}+3 a_{3}\right) \\
a_{2}\left(a_{1}+a_{2}+a_{3}\right)
\end{array}\right) .
$$

From (1), we see that $c \equiv \pm(0,0,1), \pm(1,1,1) \bmod 3$. If we assume that $c \equiv(0,0, \epsilon)$ $\bmod 3$, where $\epsilon= \pm 1$, then (2) reduces to

$$
d\left(\begin{array}{c}
0 \\
0 \\
\epsilon a_{2}
\end{array}\right) \equiv\left(\begin{array}{c}
a_{1}\left(a_{1}+2 a_{2}\right) \\
a_{2}\left(a_{1}+2 a_{2}\right) \\
a_{2}\left(a_{1}+a_{2}+a_{3}\right)
\end{array}\right) \bmod 3
$$

If $a_{1} \equiv 0 \bmod 3$, one has $2 a_{2}^{2} \equiv 0 \bmod 3$. This is a contradiction since $a$ and $c$ are linearly independent modulo 3 , and thus $a_{1} \neq \equiv 0 \bmod 3$. By an analogous argument, we see that $a_{2} \not \equiv 0 \bmod 3$, implying $a_{1} \equiv a_{2} \bmod 3$ by $a_{1}+2 a_{2} \equiv 0 \bmod 3$.

- In the case that $d \equiv 0 \bmod 3, a \equiv \pm(1,1,1) \bmod 3$. Then we have $\phi(X-Y) \equiv$ $\left(b_{1}-b_{2}\right) Y \bmod 3$, but this is a contradiction since $(X-Y)^{2} \equiv 0 \bmod 3$ in $A_{i}$ and $Y^{2} \not \equiv 0 \bmod 3$ in $A_{d}$.

- In the case that $d \equiv 1 \bmod 3, \phi(X(X+2 Y)) \equiv a_{1}\left(b_{1}+2 b_{2}\right) X Y \bmod \left(\mathcal{I}_{d}^{4}, 3\right)$, but $a_{1}\left(b_{1}+2 b_{2}\right) X Y \notin\left(\mathcal{I}_{d}^{4}, 3\right)$. This is a contradiction.

- In the case that $d \equiv 2$, there exists no isomorphism $\phi$ since $A_{i} \bmod 3$ has a homogeneous nonzero element $\alpha$ of degree two such that $\alpha^{2}=0$ but $A_{d} \bmod 3$ does not. 
Proposition 5.6 $A_{i}$ is not isomorphic to $A_{d}$ for $i=1,2$ and $d \geq 3$.

Proof From (1) and the previous lemma, we obtain $c= \pm(1,-2,1)$. Considering $-\phi$ instead of $\phi$ if necessary, we can assume $c=(1,-2,1)$. Then (2) reduces to

$$
d\left(\begin{array}{c}
a_{1}+2 a_{2}+3 a_{3} \\
-2\left(a_{1}+2 a_{2}+3 a_{3}\right) \\
-2\left(a_{1}+a_{2}+a_{3}\right)
\end{array}\right)=\left(\begin{array}{c}
a_{1}\left(a_{1}+2 a_{2}+3 a_{3}\right) \\
a_{2}\left(a_{1}+2 a_{2}+3 a_{3}\right) \\
a_{2}\left(a_{1}+a_{2}+a_{3}\right)
\end{array}\right) .
$$

Since $a$ and $c$ are linearly independent, we have $a_{1}+2 a_{2}+3 a_{3} \neq 0$ or $a_{1}+a_{2}+a_{3} \neq 0$.

- If $a_{1}+2 a_{2}+3 a_{3} \neq 0$, we obtain $a_{1}=d$ and $a_{2}=-2 d$. Then, considering the component of $Z X$ in $\phi(X(X+2 Y+3 Z)) \in A_{d}^{4}$ with respect to the basis $\left\{Z^{2}, Y Z, Z X\right\}$ of $A_{d}^{4}$, we obtain $\left(a_{3}-d\right)\left(1-d^{2}\right)=0$. Hence $a_{3}=d$ and then $a=d(1,-2,1)$, which is a contradiction.

- If $a_{1}+a_{2}+a_{3} \neq 0$, we obtain $a_{2}=-2 d$. If $a_{1}=d$, we find a contradiction in the same way as above, so we must have $a_{1}+2 a_{2}+3 a_{3}=0$. Then

$$
\phi(Y(X+2 Y+3 Z)) \equiv\left(b_{1}+2 b_{2}+3 b_{3}\right)\left(b_{2}-2\right)(1-d) Y Z \bmod \mathcal{I}_{d}^{4},
$$

so $b_{2}=2$ and $\left(a_{2}, b_{2}, c_{2}\right)=2(-d, 1,-1)$, which is a contradiction.

Proposition 5.7 For any $d, d^{\prime} \geq 3, A_{d}$ and $A_{d^{\prime}}$ are isomorphic if and only if $d=d^{\prime}$.

Proof Clearly, we only have to consider the case $d<d^{\prime}$ and prove that there exists no graded ring isomorphism from $A_{d}$ to $A_{d^{\prime}}$. Let us assume that there exists a graded ring isomorphism $\phi: A_{d} \rightarrow A_{d^{\prime}}$ given by

$$
\left(\begin{array}{l}
\phi(X) \\
\phi(Y) \\
\phi(Z)
\end{array}\right)=\left(\begin{array}{lll}
a_{1} & b_{1} & c_{1} \\
a_{2} & b_{2} & c_{2} \\
a_{3} & b_{3} & c_{3}
\end{array}\right)\left(\begin{array}{l}
X \\
Y \\
Z
\end{array}\right)
$$

and put $a=\left(a_{1}, a_{2}, a_{3}\right), b=\left(b_{1}, b_{2}, b_{3}\right), c=\left(c_{1}, c_{2}, c_{3}\right)$. From $\phi(Y(X+Z))=$ $\phi(X(X+Y+d Z))=\phi(Y(X+Y+d Z))=0$ in $A_{d^{\prime}}$, we obtain

$$
\begin{aligned}
& 0=c_{2}\left(c_{1}+c_{3}\right), \\
& 0=a_{2}\left(c_{1}+c_{3}\right)+c_{2}\left(a_{1}+a_{3}\right)-d^{\prime} a_{2}\left(a_{1}+a_{3}\right), \\
& 0=c_{1}\left(c_{1}+c_{2}+d c_{3}\right), \\
& 0=a_{1}\left(c_{1}+c_{2}+d c_{3}\right)+c_{1}\left(a_{1}+a_{2}+d a_{3}\right)-d^{\prime} a_{1}\left(a_{1}+a_{2}+d a_{3}\right), \\
& 0=c_{2}\left(c_{1}+c_{2}+d c_{3}\right), \\
& 0=a_{2}\left(c_{1}+c_{2}+d c_{3}\right)+c_{2}\left(a_{1}+a_{2}+d a_{3}\right)-d^{\prime} a_{2}\left(a_{1}+a_{2}+d a_{3}\right) .
\end{aligned}
$$


If we assume $c_{2} \neq 0$, we have $c_{1}+c_{3}=c_{1}+c_{2}+d c_{3}=0$, so $c= \pm(-1,1-d, 1)$. If $a_{1}+a_{2}+d a_{3} \neq 0$, then $d^{\prime} a_{1}= \pm 1$, which is a contradiction. Hence $a_{1}+a_{2}+d a_{3}=0$. Similarly, $a_{1}+a_{3}=0$. However, these equations contradict the linear independence of $a$ and $c$. Therefore, we obtain $c_{2}=0$ and $c_{1}\left(c_{1}+d c_{3}\right)=0$.

If $c_{1}=0$, then we have $c= \pm(0,0,1)$. In a similar way as above, we can find a contradiction if $a_{1} \neq 0$ or $a_{2} \neq 0$. Hence we obtain $a= \pm(0,0,1)$, but this contradicts the linear independence of $a$ and $c$. If $c_{1}+d c_{3}=0$, we find a contradiction in the same manner.

Proposition 5.8 $M_{1}$ and $M_{2}$ are homeomorphic.

To prove this proposition, we use Jupp's classification theorem for closed, oriented, oneconnected 6-manifolds with torsion-free cohomology [6]. While there is a complete classification of such 6-manifolds, to prove Proposition 5.8 we only need the following special case.

Theorem 5.9 [6] Let $M, N$ be closed, one-connected, smooth 6-manifolds with torsion-free cohomology. If a graded ring isomorphism $\phi: H^{*}(N ; \mathbb{Z}) \rightarrow H^{*}(M ; \mathbb{Z})$ preserves the second Stiefel-Whitney classes and the first Pontrjagin classes, then there exists a homeomorphism $f: M \rightarrow N$ which induces $\phi$ in cohomology.

Proof of Proposition 5.8 Let us define a graded ring automorphism $\phi$ of $\mathbb{Z}[X, Y, Z]$ by

$$
\left(\begin{array}{l}
\phi(X) \\
\phi(Y) \\
\phi(Z)
\end{array}\right)=\left(\begin{array}{rrr}
1 & 1 & 0 \\
0 & -1 & 0 \\
0 & 1 & 1
\end{array}\right)\left(\begin{array}{l}
X \\
Y \\
Z
\end{array}\right)
$$

We easily see that $\phi\left(\mathcal{I}_{1}^{4}+\mathcal{I}_{1}^{6}\right) \subseteq\left(\mathcal{I}_{2}^{4}+\mathcal{I}_{2}^{6}\right)$, and then $\phi$ descends to a graded ring isomorphism from $A_{1}$ to $A_{2}$. Using Theorem 2.24, we also see that $\phi$ maps the second Stiefel-Whitney class and the first Pontrjagin class of $M_{1}$ to those of $M_{2}$. Hence, by Theorem 5.9, there exists a homeomorphism between $M_{1}$ and $M_{2}$ which induces $\phi$ in cohomology.

Next, let $\lambda$ be an indecomposable characteristic matrix on $C^{3}(6)^{*}$ and $\lambda^{\prime}$ a decomposable one.

Proposition 5.10 $H^{*}(\lambda)$ and $H^{*}\left(\lambda^{\prime}\right)$ are not isomorphic as graded rings. 
Proof First, we consider the case $H^{*}(\lambda)=A_{i}$ for $i=1$, 2. If we assume that $A_{i} \cong$ $H^{*}\left(\lambda^{\prime}\right)$, we can take a basis $\left\{w_{1}, w_{2}, w_{3}\right\}$ of $A_{i}^{2} \cong H^{2}\left(\lambda^{\prime}\right)$ such that $w_{1} w_{2}=w_{1} w_{3}=$ 0 and $w_{1}^{3}$ spans $A_{i}^{6}$, because $\lambda^{\prime}$ is decomposable. Put $w_{j}=a_{j} X+b_{j} Y+c_{j} Z$ for $j=1,2,3$ and $V=\left\langle w_{2}, w_{3}\right\rangle$. Let us take $\left\{Y Z, Z X, Z^{2}\right\}$ as a basis of $A_{i}^{4}$. Then we have $c_{1} c_{2}=c_{1} c_{3}=0$ from the coefficients of $Z^{2}$ in the equation $w_{1} w_{2}=w_{1} w_{3}=0$.

- If $c_{1} \neq 0$, we have $c_{2}=c_{3}=0$ and $c_{1}= \pm 1$. Hence $X, Y \in V$. However, there occurs a contradiction since $0=w_{1} X \equiv\left(a_{1}+b_{1}\right) Y Z+c_{1} Z X \not \equiv 0 \bmod 3$.

- If $c_{1}=0$, we obtain $a_{1}\left(c_{2}-3 a_{2}\right)=a_{1}\left(c_{3}-3 a_{3}\right)=0$ from the coefficients of $Z X$ in $w_{1} w_{2}=w_{1} w_{3}=0$. Since $\left(c_{1}, c_{2}, c_{3}\right)$ is primitive, we obtain $a_{1}=0$, and then $w_{1}= \pm Y$. However, $Y^{3}=-2 Y^{2} Z$ in $A_{i}$, so this contradicts the assumption that $w_{1}^{3}$ spans $A_{i}^{6}$.

Next, we consider the case $H^{*}(\lambda)=A_{d}$ for $d \geq 3$. Let us assume that there exists a basis $\left\{w_{1}, w_{2}, w_{3}\right\}$ of $A_{d}^{2}$ as above and put again $w_{j}=a_{j} X+b_{j} Y+c_{j} Z, V=$ $\left\langle w_{2}, w_{3}\right\rangle$. Then similarly $c_{1} c_{2}=c_{1} c_{3}=0$.

- If $c_{1} \neq 0$, we obtain $c_{2}=c_{3}=0, c_{1}= \pm 1$ and $X, Y \in V$. Then we have the equation $w_{1} X=\left(a_{1}-b_{1}\right) Y Z+\left(c_{1}-d a_{1}\right) Z X=0$, but this is a contradiction since $d a_{1} \neq c_{1}= \pm 1$.

- If $c_{1}=0$, we obtain $a_{1}=0$ in the same way as for $A_{i}(i=1,2)$. However, $Y^{3}=(1-d) Y^{2} Z$ in $A_{d}$, so this also contradicts the assumption.

Finally, we show that two decomposable quasitoric manifolds over $C^{3}(6)^{*}$ are homeomorphic if their cohomology rings are isomorphic, using the cohomological rigidity of quasitoric manifolds over $C^{3}(5)^{*}=\Delta^{1} \times \Delta^{2}$. As remarked in Section 1 , this rigidity has been shown by Choi, Park and Suh [3].

Proposition 5.11 Let $M=M_{1} \# M_{2}$ and $N=N_{1} \# N_{2}$, where $M_{1}, N_{1}$ are quasitoric manifolds over $C^{3}(4)^{*}$ and $M_{2}, N_{2}$ over $C^{3}(5)^{*}$. If the cohomology rings of $M$ and $N$ are isomorphic as graded rings, then $M$ and $N$ are homeomorphic.

Proof Note that $M_{1}$ and $N_{1}$ are homeomorphic to $\mathbb{C P}^{3}$. We can naturally consider $H^{2}(M)=H^{2}\left(M_{1}\right) \oplus H^{2}\left(M_{2}\right)$. Then take a basis $\left\{u_{1}, u_{2}, u_{3}\right\}$ of $H^{2}(M)$ such that $u_{1}$ is a basis of $H^{2}\left(M_{1}\right)$ and $\left\{u_{2}, u_{3}\right\}$ is a basis of $H^{2}\left(M_{2}\right)$. We take a basis $\left\{v_{1}, v_{2}, v_{3}\right\}$ of $H^{2}(N)$ in a similar way. Assume that there exists a graded ring isomorphism $\phi: H^{*}(M) \rightarrow H^{*}(N)$, and write $\phi\left(u_{i}\right)=a_{i} v_{1}+b_{i} v_{2}+c_{i} v_{3}$ for $i=1,2,3$. Then the matrix 


$$
\left(\begin{array}{lll}
a_{1} & b_{1} & c_{1} \\
a_{2} & b_{2} & c_{2} \\
a_{3} & b_{3} & c_{3}
\end{array}\right)
$$

is invertible.

- If $a_{1}=0$, then from $u_{1} u_{2}=u_{1} u_{3}=0$ and $v_{1} v_{2}=v_{1} v_{3}=0$ we have that $\left(b_{1} v_{2}+c_{1} v_{3}\right)\left(b_{2} v_{2}+c_{2} v_{3}\right)=\left(b_{1} v_{2}+c_{1} v_{3}\right)\left(b_{3} v_{2}+c_{3} v_{3}\right)=0$. Since $b_{1} v_{2}+c_{1} v_{3} \neq 0$, $b_{2} v_{2}+c_{2} v_{3}$ and $b_{3} v_{2}+c_{3} v_{3}$ are linearly dependent. Therefore, we can take $B \in$ $\mathrm{GL}(2, \mathbb{Z})$ such that

$$
B\left(\begin{array}{lll}
a_{2} & b_{2} & c_{2} \\
a_{3} & b_{3} & c_{3}
\end{array}\right)=\left(\begin{array}{ccc}
0 & b_{2}^{\prime} & c_{2}^{\prime} \\
1 & 0 & 0
\end{array}\right) .
$$

(Notice that $b_{2} v_{2}+c_{2} v_{3}$ and $b_{3} v_{2}+c_{3} v_{3}$ are linearly dependent, but $a_{2} v_{1}+b_{2} v_{2}+c_{2} v_{3}$ and $a_{3} v_{1}+b_{3} v_{2}+c_{3} v_{3}$ are linearly independent.) Then we see that $v_{2}^{\prime}:=b_{1} v_{2}+c_{1} v_{3}$ and $v_{3}^{\prime}:=b_{2}^{\prime} v_{2}+c_{2}^{\prime} v_{3}$ form a basis of $H^{2}\left(N_{2}\right)$. Moreover, since $v_{2}^{\prime}=\phi\left(u_{1}\right)$ and $v_{3}^{\prime}$ is a linear combination of $\phi\left(u_{2}\right)$ and $\phi\left(u_{3}\right)$, we have $v_{2}^{\prime} v_{3}^{\prime}=0$. Since quasitoric manifolds over $C^{3}(5)^{*}$ are cohomologically rigid, $N_{2}$ is homeomorphic to $\mathbb{C P}^{3} \# \mathbb{C P}^{3}$, and therefore $N$ is homeomorphic to $\mathbb{C P} \mathrm{P}^{3} \# \mathbb{C P} \mathbb{P}^{3} \# \mathbb{C P}$.

Similarly, taking $\left(u_{2}^{\prime}, u_{3}^{\prime}\right)=\phi^{-1}\left(v_{3}^{\prime}, v_{1}\right), u_{2}^{\prime}$ and $u_{3}^{\prime}$ are in $H^{2}\left(M_{2}\right)$ since $u_{1} u_{2}^{\prime}=$ $\phi^{-1}\left(v_{2}^{\prime} v_{3}^{\prime}\right)=0, u_{1} u_{3}^{\prime}=\phi^{-1}\left(v_{2}^{\prime} v_{1}\right)=0$. Thus $\left\{u_{2}^{\prime}, u_{3}^{\prime}\right\}$ is a basis of $H^{2}\left(M_{2}\right)$ and $u_{2}^{\prime} u_{3}^{\prime}=0$, so $M$ is also homeomorphic to $\mathbb{C P}^{3} \# \mathbb{C P}^{3} \# \mathbb{C P}$.

- If $a_{1} \neq 0$, similarly we have $a_{2}=a_{3}=0$. Then we see that the correspondence $u_{2} \mapsto b_{2} v_{2}+c_{2} v_{3}, u_{3} \mapsto b_{3} v_{2}+c_{3} v_{3}$ provides an isomorphism $H^{*}\left(M_{2}\right) \rightarrow H^{*}\left(N_{2}\right)$. Therefore, from the cohomological rigidity of quasitoric manifolds over $C^{3}(5)^{*}, M_{2}$ is homeomorphic to $N_{2}$. Since $M_{1}=N_{1}=\mathbb{C P}^{3}, M$ and $N$ are also homeomorphic.

Then we see that $M$ is homeomorphic to $N$ in each case.

We obtain the following theorem.

Theorem 5.12 Over $C^{3}(6)^{*}$, there exist countably infinite quasitoric manifolds up to homeomorphism and they are distinguished by their integral cohomology rings.

The classification of quasitoric manifolds over $C^{3}(6)^{*}$ is comparatively easy since indecomposable characteristic matrices are almost parametrized by one integer $d$, but it is more complicated for $C^{3}(m)^{*}$ with $m>6$. There are countably infinite indecomposable characteristic matrices on $C^{3}(m)^{*}$ up to equivalence, and for large $m$, it seems hard to list them all. Then, for the time being, let us consider the classification of small covers over $C^{3}(m)^{*}$. 
Lemma 5.13 For $m>5$, any real characteristic matrix on $C^{3}(m)^{*}$ is decomposable.

Proof Let $\lambda$ be a real characteristic matrix on $C^{3}(m)^{*}$ for $m>5$. We can assume that $\lambda$ is of the form

$$
\lambda=\left(\begin{array}{ccccccc}
1 & 0 & 0 & a_{1} & b_{1} & \cdots & 1 \\
0 & 1 & 0 & 1 & b_{2} & \cdots & c_{2} \\
0 & 0 & 1 & a_{3} & b_{3} & \cdots & 1
\end{array}\right),
$$

since $|1,2,3|=|1,3,4|=|1,2, m|=|2,3, m|=1$. If $c_{2}=1$, then $|1,3, m|=1$, so $\lambda$ is decomposable. Otherwise, if $c_{2}=0$, then $|1,4, m|=1$. Hence $\lambda$ is decomposable.

Then we list all indecomposable real characteristic matrices over $C^{3}(m)^{*}$ for $m=4,5$ up to equivalence:

- On $C^{3}(4)^{*}$, there exists only one real characteristic matrix

$$
\lambda=\left(\begin{array}{llll}
1 & 0 & 0 & 1 \\
0 & 1 & 0 & 1 \\
0 & 0 & 1 & 1
\end{array}\right)
$$

up to equivalence, and the corresponding small cover is $\mathbb{R} \mathrm{P}^{3}$.

- On $C^{3}(5)^{*}=\Delta^{1} \times \Delta^{2}$, there exist two indecomposable real characteristic matrices up to equivalence,

$$
\lambda_{1}=\left(\begin{array}{ll|lll}
1 & 1 & 0 & 0 & 0 \\
\hline 0 & 0 & 1 & 0 & 1 \\
0 & 0 & 0 & 1 & 1
\end{array}\right) \quad \text { and } \quad \lambda_{2}=\left(\begin{array}{ll|lll}
1 & 1 & 0 & 0 & 0 \\
\hline 0 & 1 & 1 & 0 & 1 \\
0 & 1 & 0 & 1 & 1
\end{array}\right),
$$

where we label the facets $F_{1}, \ldots, F_{5}$ so that $F_{1}, F_{2}$ correspond to the facets of $\Delta^{1}$ and $F_{3}, F_{4}, F_{5}$ to those of $\Delta^{2}$.

Using the real moment-angle manifold $\mathbb{R}_{\Delta^{1} \times \Delta^{2}}=S^{1} \times S^{2}$, we easily see that $\mathbb{R} M\left(\lambda_{1}\right)$ is homeomorphic to $\mathbb{R} P^{1} \times \mathbb{R} \mathrm{P}^{2}$. Moreover, we can construct a homeomorphism from $\mathbb{R} M\left(\lambda_{2}\right)$ to $\mathbb{R} M\left(\lambda_{1}\right)$ explicitly, as the map induced by

$$
\begin{aligned}
S^{1} \times S^{2} & \rightarrow S^{1} \times S^{2}, \\
\left(z_{1}, z_{2}, w_{1}, w_{2}, w_{3}\right) & \mapsto\left(z_{1}, z_{2}, z_{1} w_{1}+z_{2} w_{2},-z_{2} w_{1}+z_{1} w_{2}, w_{3}\right),
\end{aligned}
$$

which is a weakly equivariant homeomorphism with respect to the free action of $S_{\lambda_{2}}$ on the domain, and that of $S_{\lambda_{1}}$ on the codomain. Here each $S_{\lambda_{i}}$ denotes the kernel of $\lambda_{i}:(\mathbb{Z} / 2)^{5} \rightarrow(\mathbb{Z} / 2)^{3}$ for $i=1,2$. This construction follows the one for quasitoric manifolds over $\Delta^{1} \times \Delta^{n}$ by Choi, Park and Suh [3]. 
Then we obtain the topological classification of small covers over $C^{3}(m)^{*}$.

Theorem 5.14 For any $m>3$, a small cover over $C^{3}(m)^{*}$ is homeomorphic to the connected sum of copies of $\mathbb{R} \mathrm{P}^{3}$ and $\mathbb{R P}^{1} \times \mathbb{R} \mathrm{P}^{2}$.

\section{Small covers of higher dimensions}

In this section, we list all real characteristic matrices over the dual cyclic polytopes of dimension $n \geq 4$. As noted in Section 1, we only consider the case $m-n \geq 3$, where $m$ denotes the number of facets. We will use the following easy lemma.

Lemma 6.1 If there exists a (real) characteristic matrix $\lambda$ on $C^{n+1}(m+1)^{*}$ of the form

$$
\lambda=\left(\begin{array}{c|ccc}
1 & a_{2} & \cdots & a_{m} \\
\hline 0 & & & \\
\vdots & & A & \\
0 & &
\end{array}\right),
$$

then $A$ is a (real) characteristic matrix on $C^{n}(m)^{*}$.

Proof It follows immediately from Gale's evenness condition: If $\left\{i_{1}, \ldots, i_{n}\right\}$ is a simplex of $K_{C^{n}(m)^{*}}$, then $\left\{1, i_{1}+1, \ldots, i_{n}+1\right\}$ is a simplex of $K_{C^{n+1}(m+1)^{*}}$. Hence we have

$$
\left|i_{1}, \ldots, i_{n}\right|_{A}=\left|1, i_{1}+1, \ldots, i_{n}+1\right|_{\lambda}= \pm 1
$$

for any $\left\{i_{1}, \ldots, i_{n}\right\} \in K_{C^{n}(m)^{*}}$.

Proposition 6.2 If $n \geq 4$ and $m-n \geq 4$, there exists no real characteristic matrix on $C^{n}(m)^{*}$.

Proof By the previous lemma, we only have to prove that there exists no real characteristic matrix on $C^{4}(m)^{*}$ if $m \geq 8$. Assume that there exists a real characteristic matrix $\lambda$ on $C^{4}(m)^{*}$ for $m \geq 8$. All the maximal faces of $K_{C^{4}(m)^{*}}$ are

$$
\begin{array}{ll}
\{1, i, i+1, m\} & (i=2,3, \ldots, m-2), \\
\{i, i+1, j, j+1\} & (1 \leq i<j-1 \leq m-2) .
\end{array}
$$

We can assume that $\lambda$ is of the form

$$
\left(\begin{array}{cccc|ccccc}
1 & 0 & 0 & 0 & 1 & b_{1} & c_{1} & \cdots & d_{1} \\
0 & 1 & 0 & 0 & a_{2} & b_{2} & c_{2} & \cdots & 1 \\
0 & 0 & 1 & 0 & 1 & b_{3} & c_{3} & \cdots & d_{3} \\
0 & 0 & 0 & 1 & a_{4} & b_{4} & c_{4} & \cdots & 1
\end{array}\right) .
$$


Let us consider the four cases $\mathbf{a}=(1,0,1,0), \mathbf{a}=(1,1,1,1), \mathbf{a}=(1,1,1,0)$ and $\mathbf{a}=(1,0,1,1)$ separately, where $\mathbf{a}:=\left(1, a_{2}, 1, a_{4}\right)$.

Case 1 If $\mathbf{a}=(1,0,1,0), \lambda$ is of the form

$$
\left(\begin{array}{cccc|ccccc}
1 & 0 & 0 & 0 & 1 & b_{1} & c_{1} & \cdots & d_{1} \\
0 & 1 & 0 & 0 & 0 & b_{2} & c_{2} & \cdots & 1 \\
0 & 0 & 1 & 0 & 1 & b_{3} & c_{3} & \cdots & d_{3} \\
0 & 0 & 0 & 1 & 0 & b_{4} & c_{4} & \cdots & 1
\end{array}\right) .
$$

From the nonsingularity condition, we have

$$
1=|1,2,5,6|=b_{4}, \quad 1=|3,4,5,6|=b_{2} .
$$

Then we have $|1,5,6, m|=0$, but this contradicts the nonsingularity condition.

Case 2 If $\mathbf{a}=(1,1,1,1), \lambda$ is of the form

$$
\left(\begin{array}{cccc|ccccc}
1 & 0 & 0 & 0 & 1 & b_{1} & c_{1} & \cdots & d_{1} \\
0 & 1 & 0 & 0 & 1 & b_{2} & c_{2} & \cdots & 1 \\
0 & 0 & 1 & 0 & 1 & b_{3} & c_{3} & \cdots & d_{3} \\
0 & 0 & 0 & 1 & 1 & b_{4} & c_{4} & \cdots & 1
\end{array}\right) .
$$

From the nonsingularity condition, we have

$$
1=|1,2,5,6|=b_{3}+b_{4}, \quad 1=|2,3,5,6|=b_{1}+b_{4}, \quad 1=|3,4,5,6|=b_{1}+b_{2} .
$$

Therefore $\mathbf{b}:=\left(b_{1}, b_{2}, b_{3}, b_{4}\right)$ is $(1,0,1,0)$ or $(0,1,0,1)$. In each case, we have the contradiction $1=|1,5,6, m|=0$.

Case 3 If $\mathbf{a}=(1,1,1,0), \lambda$ is of the form

$$
\left(\begin{array}{cccc|ccccc}
1 & 0 & 0 & 0 & 1 & b_{1} & c_{1} & \cdots & d_{1} \\
0 & 1 & 0 & 0 & 1 & b_{2} & c_{2} & \cdots & 1 \\
0 & 0 & 1 & 0 & 1 & b_{3} & c_{3} & \cdots & d_{3} \\
0 & 0 & 0 & 1 & 0 & b_{4} & c_{4} & \cdots & 1
\end{array}\right) .
$$

From the nonsingularity condition, we have $b_{4}=1, b_{1}+b_{2}=1, d_{3}=0$ and $b_{2}+b_{3}=0$. Therefore $\mathbf{b}$ is $(0,1,1,1)$ or $(1,0,0,1)$.

- In the case that $\mathbf{b}=(0,1,1,1)$, we have $1=|1,2,6,7|=c_{3}+c_{4}, 1=|2,3,6,7|=c_{1}$ and $1=|4,5,6,7|=c_{2}+c_{3}$. In particular, we have $c_{2}=c_{4}$. Then $1=|1,6,7, m|=0$, which is a contradiction.

- In the case that $\mathbf{b}=(1,0,0,1)$, we have $1=|1,2,6,7|=c_{3}, 1=|3,4,6,7|=c_{2}$, and then $1=|4,5,6,7|=0$. This is a contradiction. 
Case 4 If $\mathbf{a}=(1,0,1,1), \lambda$ is of the form

$$
\left(\begin{array}{cccc|ccccc}
1 & 0 & 0 & 0 & 1 & b_{1} & c_{1} & \cdots & d_{1} \\
0 & 1 & 0 & 0 & 0 & b_{2} & c_{2} & \cdots & 1 \\
0 & 0 & 1 & 0 & 1 & b_{3} & c_{3} & \cdots & d_{3} \\
0 & 0 & 0 & 1 & 1 & b_{4} & c_{4} & \cdots & 1
\end{array}\right) .
$$

From the nonsingularity condition, we have $b_{3}+b_{4}=1, b_{1}+b_{4}=1, b_{2}=1$, and then $d_{3}=1$. Therefore $\mathbf{b}$ is $(0,1,0,1)$ or $(1,1,1,0)$.

- In the case that $\mathbf{b}=(0,1,0,1)$, we have $1=|1,2,6,7|=c_{3}, 1=|2,3,6,7|=c_{1}$, and then $|4,5,6,7|=0$. This is a contradiction.

- In the case that $\mathbf{b}=(1,1,1,0)$, we have $1=|1,2,6,7|=c_{4}, 1=|3,4,6,7|=c_{1}+c_{2}$ and $1=|4,5,6,7|=c_{1}+c_{3}$. In particular, we have $c_{2}=c_{3}$, whence $|1,6,7, m|=0$, which is a contradiction.

Next, we will determine all real characteristic matrices on $C^{4}(7)^{*}$. Note that the maximal faces of $K_{C^{4}(7) *}$ are

$$
\begin{array}{lllll}
\{1,2,3,4\}, & \{2,3,4,5\}, & \{1,2,4,5\}, & \{1,3,4,7\}, & \{1,2,3,7\}, \\
\{1,2,5,6\}, & \{1,2,6,7\}, & \{2,3,5,6\}, & \{2,3,6,7\}, & \{3,4,5,6\}, \\
\{3,4,6,7\}, & \{1,4,5,7\}, & \{1,5,6,7\}, & \{4,5,6,7\} . &
\end{array}
$$

Proposition 6.3 $\mathbb{R}_{C^{4}(7)^{*}}$ has exactly two elements, and they are represented by the matrices

$$
\left(\begin{array}{llll|lll}
1 & 0 & 0 & 0 & 1 & 0 & 1 \\
0 & 1 & 0 & 0 & 1 & 1 & 1 \\
0 & 0 & 1 & 0 & 1 & 1 & 0 \\
0 & 0 & 0 & 1 & 0 & 1 & 1
\end{array}\right), \quad\left(\begin{array}{llll|lll}
1 & 0 & 0 & 0 & 1 & 1 & 0 \\
0 & 1 & 0 & 0 & 0 & 1 & 1 \\
0 & 0 & 1 & 0 & 1 & 1 & 1 \\
0 & 0 & 0 & 1 & 1 & 0 & 1
\end{array}\right) .
$$

Proof It is straightforward to check that the above two matrices are real characteristic matrices on $C^{4}(7)^{*}$. Then we prove that there exists no other real characteristic matrix on $C^{4}(7)^{*}$ up to the action of $\mathrm{GL}(4, \mathbb{Z} / 2)$. As in the proof of the previous proposition, we can write a real characteristic matrix on $C^{4}(7)^{*}$ of the form

$$
\lambda=\left(\begin{array}{cccc|ccc}
1 & 0 & 0 & 0 & 1 & b_{1} & c_{1} \\
0 & 1 & 0 & 0 & a_{2} & b_{2} & 1 \\
0 & 0 & 1 & 0 & 1 & b_{3} & c_{3} \\
0 & 0 & 0 & 1 & a_{4} & b_{4} & 1
\end{array}\right) .
$$


We consider again the cases $\mathbf{a}=(1,0,1,0), \mathbf{a}=(1,1,1,1), \mathbf{a}=(1,1,1,0)$ and $\mathbf{a}=(1,0,1,1)$ separately, where $\mathbf{a}:=\left(1, a_{2}, 1, a_{4}\right)$. We can see that the first two cases lead to contradictions by setting $m=7$ in the previous proof.

Case 3 If $\mathbf{a}=(1,1,1,0), \lambda$ is of the form

$$
\left(\begin{array}{cccc|ccc}
1 & 0 & 0 & 0 & 1 & b_{1} & c_{1} \\
0 & 1 & 0 & 0 & 1 & b_{2} & 1 \\
0 & 0 & 1 & 0 & 1 & b_{3} & c_{3} \\
0 & 0 & 0 & 1 & 0 & b_{4} & 1
\end{array}\right) .
$$

From the nonsingularity condition, we have

$$
\begin{array}{ll}
1=|1,2,5,6|=b_{4}, & 1=|3,4,5,6|=b_{1}+b_{2}, \quad 1=|1,4,5,7|=c_{3}+1, \\
1=|1,5,6,7|=b_{2}+b_{3}+1, & 1=|1,2,6,7|=b_{3} .
\end{array}
$$

Therefore $\mathbf{b}:=\left(b_{1}, b_{2}, b_{3}, b_{4}\right)$ is $(0,1,1,1)$. We also have $c_{1}=|2,3,6,7|=1$, so $\lambda$ is equal to the left matrix in the statement.

Case 4 If $\mathbf{a}=(1,0,1,1), \lambda$ is of the form

$$
\left(\begin{array}{cccc|ccc}
1 & 0 & 0 & 0 & 1 & b_{1} & c_{1} \\
0 & 1 & 0 & 0 & 0 & b_{2} & 1 \\
0 & 0 & 1 & 0 & 1 & b_{3} & c_{3} \\
0 & 0 & 0 & 1 & 1 & b_{4} & 1
\end{array}\right) .
$$

From the nonsingularity condition, we have

$$
\begin{array}{ll}
1=|1,2,5,6|=b_{3}+b_{4}, & 1=|2,3,5,6|=b_{1}+b_{4}, \quad 1=|3,4,5,6|=b_{2}, \\
1=|1,5,6,7|=c_{3}, & 1=|4,5,6,7|=c_{1}+1 .
\end{array}
$$

Therefore $\mathbf{c}:=\left(c_{1}, c_{2}, c_{3}, c_{4}\right)$ is $(0,1,1,1)$. We also have $b_{1}=|3,4,6,7|=1$, so $\lambda$ is equal to the right matrix in the statement.

Corollary 6.4 $\mathbb{R}_{C^{5}(8) *}$ has exactly two elements, and they are represented by the matrices

$$
\left(\begin{array}{lllll|lll}
1 & 0 & 0 & 0 & 0 & 0 & 0 & 1 \\
0 & 1 & 0 & 0 & 0 & 1 & 0 & 1 \\
0 & 0 & 1 & 0 & 0 & 1 & 1 & 1 \\
0 & 0 & 0 & 1 & 0 & 1 & 1 & 0 \\
0 & 0 & 0 & 0 & 1 & 0 & 1 & 1
\end{array}\right), \quad\left(\begin{array}{lllll|lll}
1 & 0 & 0 & 0 & 0 & 0 & 0 & 1 \\
0 & 1 & 0 & 0 & 0 & 1 & 1 & 0 \\
0 & 0 & 1 & 0 & 0 & 0 & 1 & 1 \\
0 & 0 & 0 & 1 & 0 & 1 & 1 & 1 \\
0 & 0 & 0 & 0 & 1 & 1 & 0 & 1
\end{array}\right) .
$$


Proof We can easily check that the above two matrices satisfy the nonsingularity condition for $C^{5}(8)^{*}$. Let $\lambda$ be a real characteristic matrix on $C^{5}(8)^{*}$. By Lemma 6.1, we can assume that $\lambda$ is of the form

$$
\left(\begin{array}{l|llll|lll}
1 & 0 & 0 & 0 & 0 & a & b & 1 \\
\hline 0 & 1 & 0 & 0 & 0 & 1 & 0 & 1 \\
0 & 0 & 1 & 0 & 0 & 1 & 1 & 1 \\
0 & 0 & 0 & 1 & 0 & 1 & 1 & 0 \\
0 & 0 & 0 & 0 & 1 & 0 & 1 & 1
\end{array}\right) \quad \text { or } \quad\left(\begin{array}{c|cccc|ccc}
1 & 0 & 0 & 0 & 0 & a & b & 1 \\
\hline 0 & 1 & 0 & 0 & 0 & 1 & 1 & 0 \\
0 & 0 & 1 & 0 & 0 & 0 & 1 & 1 \\
0 & 0 & 0 & 1 & 0 & 1 & 1 & 1 \\
0 & 0 & 0 & 0 & 1 & 1 & 0 & 1
\end{array}\right) \text {. }
$$

In the former case, the nonsingularity condition provides

$$
1=|2,3,6,7,8|=a+b+1, \quad 1=|4,5,6,7,8|=a+1 .
$$

Hence $\lambda$ is equal to the left matrix in the statement.

In the latter case, the nonsingularity condition provides

$$
1=|2,3,6,7,8|=a+1, \quad 1=|3,4,6,7,8|=a+b+1 .
$$

Hence $\lambda$ is equal to the right matrix in the statement.

For any matrix of the form

$$
\left(\begin{array}{c|ccccc|ccc}
1 & 0 & 0 & 0 & 0 & 0 & 1 & a & b \\
\hline 0 & 1 & 0 & 0 & 0 & 0 & 0 & 0 & 1 \\
0 & 0 & 1 & 0 & 0 & 0 & 1 & 0 & 1 \\
0 & 0 & 0 & 1 & 0 & 0 & 1 & 1 & 1 \\
0 & 0 & 0 & 0 & 1 & 0 & 1 & 1 & 0 \\
0 & 0 & 0 & 0 & 0 & 1 & 0 & 1 & 1
\end{array}\right) \quad \text { or } \quad\left(\begin{array}{c|ccccc|ccc}
1 & 0 & 0 & 0 & 0 & 0 & 1 & a & b \\
\hline 0 & 1 & 0 & 0 & 0 & 0 & 0 & 0 & 1 \\
0 & 0 & 1 & 0 & 0 & 0 & 1 & 1 & 0 \\
0 & 0 & 0 & 1 & 0 & 0 & 0 & 1 & 1 \\
0 & 0 & 0 & 0 & 1 & 0 & 1 & 1 & 1 \\
0 & 0 & 0 & 0 & 0 & 1 & 1 & 0 & 1
\end{array}\right) \text {, }
$$

it is obvious that $|3,4,5,6,7,8|=0$. Then we have the following corollary.

Corollary 6.5 There exists no real characteristic matrix on $C^{n}(n+3)^{*}$ for $n>5$.

\section{Classification for $C^{4}(7)^{*}$}

In the previous section, we showed that there are exactly two real characteristic matrices on $C^{4}(7)^{*}$ up to the left $\operatorname{GL}(4, \mathbb{Z} / 2)$-action, and they are represented by the matrices

$$
\bar{\lambda}=\left(\begin{array}{llll|lll}
1 & 0 & 0 & 0 & 1 & 0 & 1 \\
0 & 1 & 0 & 0 & 1 & 1 & 1 \\
0 & 0 & 1 & 0 & 1 & 1 & 0 \\
0 & 0 & 0 & 1 & 0 & 1 & 1
\end{array}\right), \quad \bar{\lambda}^{\prime}=\left(\begin{array}{llll|lll}
1 & 0 & 0 & 0 & 1 & 1 & 0 \\
0 & 1 & 0 & 0 & 0 & 1 & 1 \\
0 & 0 & 1 & 0 & 1 & 1 & 1 \\
0 & 0 & 0 & 1 & 1 & 0 & 1
\end{array}\right) .
$$


In a way similar to the proof of Lemma 5.3, it is shown that Aut $\left(K_{C^{4}(7)^{*}}\right)$ is generated by

$$
\sigma=\left(\begin{array}{lllllll}
1 & 2 & 3 & 4 & 5 & 6 & 7 \\
2 & 3 & 4 & 5 & 6 & 7 & 1
\end{array}\right) \quad \text { and } \quad \tau=\left(\begin{array}{lllllll}
1 & 2 & 3 & 4 & 5 & 6 & 7 \\
7 & 6 & 5 & 4 & 3 & 2 & 1
\end{array}\right)
$$

Note that Aut $\left(K_{C^{4}(7)^{*}}\right)$ acts on $\mathbb{R}_{C^{4}(7)^{*}}$ by $\sigma(\bar{\lambda})=\bar{\lambda}, \sigma\left(\bar{\lambda}^{\prime}\right)=\bar{\lambda}^{\prime}$ and $\tau(\bar{\lambda})=\bar{\lambda}^{\prime}$. Then we have the following proposition.

Proposition 7.1 There exists only one small cover over $C^{4}(7)^{*}$ up to weakly equivariant homeomorphism.

Let $p$ denote the map $\mathcal{X}_{C^{4}(7)^{*}} \rightarrow \mathbb{R}_{C^{4}(7)^{*}}$ induced from the modulo 2 reduction. We see that $p^{-1}(\bar{\lambda}) /\langle\sigma\rangle$ corresponds to $\mathcal{X}_{C^{4}(7)^{*}} / \operatorname{Aut}\left(K_{C^{4}(7)^{*}}\right)$, so first we have to list all elements of $p^{-1}(\bar{\lambda})$.

Proposition 7.2 There are exactly twenty-eight elements in $p^{-1}(\bar{\lambda})$, which are represented by the characteristic matrices

$$
\begin{aligned}
& \lambda_{1}=\left(\begin{array}{rrr}
1 & 0 & -1 \\
-1 & 1 & 1 \\
1 & 1 & 0 \\
0 & 1 & 1
\end{array}\right), \quad \lambda_{2}=\left(\begin{array}{lll}
1 & 2 & 1 \\
1 & 1 & 1 \\
1 & 1 & 0 \\
0 & 1 & 1
\end{array}\right), \quad \lambda_{3}=\left(\begin{array}{lll}
1 & 0 & 1 \\
1 & 1 & 1 \\
1 & 1 & 0 \\
2 & 1 & 1
\end{array}\right), \quad \lambda_{4}=\left(\begin{array}{lll}
1 & 2 & 1 \\
1 & 3 & 1 \\
1 & 1 & 0 \\
2 & 3 & 1
\end{array}\right), \\
& \lambda_{5}=\left(\begin{array}{ccc}
1 & 0 & 1 \\
1 & 1 & 1 \\
1 & 1 & 0 \\
0 & -1 & 1
\end{array}\right), \quad \lambda_{6}=\left(\begin{array}{lll}
1 & 2 & 1 \\
1 & 3 & 1 \\
1 & 1 & 0 \\
0 & 1 & 1
\end{array}\right), \quad \lambda_{7}=\left(\begin{array}{rrr}
1 & 0 & 1 \\
1 & -1 & 1 \\
1 & 1 & 0 \\
2 & 1 & 1
\end{array}\right), \quad \lambda_{8}=\left(\begin{array}{lll}
1 & 2 & 1 \\
1 & 1 & 1 \\
1 & 1 & 0 \\
2 & 3 & 1
\end{array}\right) \text {, } \\
& \lambda_{9}=\left(\begin{array}{lll}
1 & 0 & 1 \\
1 & 1 & 1 \\
1 & 1 & 0 \\
0 & 1 & 1
\end{array}\right), \lambda_{10}=\left(\begin{array}{rrr}
1 & -2 & -1 \\
-1 & 1 & 1 \\
1 & 1 & 0 \\
0 & 1 & 1
\end{array}\right), \lambda_{11}=\left(\begin{array}{rrr}
1 & 0 & -1 \\
1 & 1 & 1 \\
1 & 1 & 0 \\
0 & 1 & 1
\end{array}\right), \lambda_{12}=\left(\begin{array}{lll}
1 & 0 & 1 \\
3 & 1 & 1 \\
1 & 1 & 0 \\
2 & 1 & 1
\end{array}\right) \text {, } \\
& \lambda_{13}=\left(\begin{array}{rrr}
1 & 0 & 1 \\
3 & 1 & 1 \\
1 & 1 & 0 \\
0 & -1 & 1
\end{array}\right), \lambda_{14}=\left(\begin{array}{rrr}
1 & 0 & 1 \\
-1 & -1 & 1 \\
1 & 1 & 0 \\
2 & 1 & 1
\end{array}\right), \lambda_{15}=\left(\begin{array}{rrr}
1 & 0 & -1 \\
1 & 1 & 1 \\
1 & 1 & 0 \\
0 & -1 & 1
\end{array}\right), \lambda_{16}=\left(\begin{array}{rrr}
1 & 0 & -1 \\
1 & 1 & 1 \\
1 & 1 & 0 \\
2 & 1 & 1
\end{array}\right), \\
& \lambda_{17}=\left(\begin{array}{rrr}
1 & 0 & -1 \\
-1 & -1 & 1 \\
1 & 1 & 0 \\
2 & 1 & 1
\end{array}\right), \lambda_{18}=\left(\begin{array}{lll}
1 & 2 & 3 \\
1 & 1 & 1 \\
1 & 1 & 0 \\
0 & 1 & 1
\end{array}\right), \lambda_{19}=\left(\begin{array}{lll}
1 & 0 & 1 \\
1 & 1 & 1 \\
1 & 3 & 2 \\
0 & 1 & 1
\end{array}\right), \lambda_{20}=\left(\begin{array}{lll}
1 & 2 & 1 \\
1 & 1 & 1 \\
1 & 1 & 2 \\
0 & 1 & 1
\end{array}\right) \text {, }
\end{aligned}
$$




$$
\begin{aligned}
& \lambda_{21}=\left(\begin{array}{lll}
1 & 0 & 1 \\
1 & 1 & 1 \\
1 & 1 & 2 \\
0 & 1 & 1
\end{array}\right), \lambda_{22}=\left(\begin{array}{lll}
1 & 2 & 1 \\
1 & 1 & 1 \\
1 & 3 & 2 \\
0 & 1 & 1
\end{array}\right), \lambda_{23}=\left(\begin{array}{rrr}
1 & 0 & 1 \\
1 & -1 & 1 \\
1 & 1 & 2 \\
0 & 1 & 1
\end{array}\right), \lambda_{24}=\left(\begin{array}{lll}
1 & 2 & 1 \\
1 & 3 & 1 \\
1 & 3 & 2 \\
0 & 1 & 1
\end{array}\right), \\
& \lambda_{25}=\left(\begin{array}{lll}
1 & 0 & 1 \\
1 & 1 & 1 \\
1 & 1 & 2 \\
2 & 1 & 1
\end{array}\right), \lambda_{26}=\left(\begin{array}{rrr}
1 & 0 & -1 \\
1 & 1 & 1 \\
1 & 1 & 2 \\
0 & 1 & 1
\end{array}\right), \lambda_{27}=\left(\begin{array}{rrr}
1 & 0 & -1 \\
1 & 1 & 1 \\
1 & 1 & 2 \\
2 & 1 & 1
\end{array}\right), \lambda_{28}=\left(\begin{array}{lll}
1 & 2 & 3 \\
1 & 1 & 1 \\
1 & 1 & 2 \\
0 & 1 & 1
\end{array}\right),
\end{aligned}
$$

where by a matrix $*$ we mean the whole matrix $(I \mid *)$, as in Proposition 5.4.

Proof Let $\lambda$ be a characteristic matrix on $C^{4}(7)^{*}$ of the form

$$
\lambda=\left(\begin{array}{llll|lll}
1 & 0 & 0 & 0 & a_{1} & b_{1} & c_{1} \\
0 & 1 & 0 & 0 & a_{2} & b_{2} & c_{2} \\
0 & 0 & 1 & 0 & a_{3} & b_{3} & c_{3} \\
0 & 0 & 0 & 1 & a_{4} & b_{4} & c_{4}
\end{array}\right) \equiv\left(\begin{array}{llll|lll}
1 & 0 & 0 & 0 & 1 & 0 & 1 \\
0 & 1 & 0 & 0 & 1 & 1 & 1 \\
0 & 0 & 1 & 0 & 1 & 1 & 0 \\
0 & 0 & 0 & 1 & 0 & 1 & 1
\end{array}\right) \bmod 2 .
$$

Then $|2,3,4,5|_{\lambda}=-a_{1}= \pm 1,|1,2,4,5|_{\lambda}=-a_{3}= \pm 1,|1,3,4,7|_{\lambda}=c_{2}= \pm 1$ and $|1,2,3,7|_{\lambda}=c_{4}= \pm 1$, so we can assume

$$
\lambda=\left(\begin{array}{cccc|ccc}
1 & 0 & 0 & 0 & 1 & b_{1} & c_{1} \\
0 & 1 & 0 & 0 & a_{2} & b_{2} & 1 \\
0 & 0 & 1 & 0 & 1 & b_{3} & c_{3} \\
0 & 0 & 0 & 1 & a_{4} & b_{4} & 1
\end{array}\right)
$$

up to the action of $\operatorname{GL}(4, \mathbb{Z})$ and $(\mathbb{Z} / 2)^{7}$. The nonsingularity condition for $\lambda$ is equivalent to the equations

$$
\begin{aligned}
|1,2,5,6|_{\lambda} & =b_{4}-a_{4} b_{3}= \pm 1, \\
|1,2,6,7|_{\lambda} & =b_{3}-b_{4} c_{3}= \pm 1, \\
|2,3,5,6|_{\lambda} & =b_{4}-a_{4} b_{1}= \pm 1 \\
|2,3,6,7|_{\lambda} & =b_{1}-b_{4} c_{1}= \pm 1 \\
|3,4,5,6|_{\lambda} & =b_{2}-a_{2} b_{1}= \pm 1, \\
|3,4,6,7|_{\lambda} & =b_{1}-b_{2} c_{1}= \pm 1, \\
|1,4,5,7|_{\lambda} & =a_{2} c_{3}-1= \pm 1, \\
|1,5,6,7|_{\lambda} & =\left|\begin{array}{ccc}
a_{2} & b_{2} & 1 \\
1 & b_{3} & c_{3} \\
a_{4} & b_{4} & 1
\end{array}\right|= \pm 1,
\end{aligned}
$$




$$
|4,5,6,7|_{\lambda}=-\left|\begin{array}{ccc}
1 & b_{1} & c_{1} \\
a_{2} & b_{2} & 1 \\
1 & b_{3} & c_{3}
\end{array}\right|= \pm 1 .
$$

Up to the action of $(\mathbb{Z} / 2)^{7}$, we can assume $b_{3}>0$.

(i) First, we consider the case $|1,4,5,7|_{\lambda}=a_{2} c_{3}-1=-1$, namely $c_{3}=0$ (since $\left.a_{2} \equiv 1 \bmod 2\right)$. From (4), we obtain $b_{3}=1$. From (6)-(8), $\left(b_{2}-b_{4}\right) c_{1}=0, \pm 2$. Similarly, we obtain $a_{4}\left(b_{1}-1\right)=0, \pm 2$ from (3)-(5).

(i-A) If $\left(b_{2}-b_{4}\right) c_{1}=0$ and $a_{4}\left(b_{1}-1\right)=0$, we obtain $b_{2}=b_{4}\left(\right.$ since $\left.c_{1} \equiv 1 \bmod 2\right)$ and $a_{4}=0$ (since $\left.b_{1} \equiv 0 \bmod 2\right)$. From (3), $b_{4}= \pm 1$. Similarly, from (10), we obtain $a_{2}= \pm 1$. Since $\lambda$ coincides with the matrix

$$
\left(\begin{array}{rrrr|rcr}
1 & 0 & 0 & 0 & 1 & b_{1} & -c_{1} \\
0 & 1 & 0 & 0 & -a_{2} & -b_{4} & 1 \\
0 & 0 & 1 & 0 & 1 & 1 & 0 \\
0 & 0 & 0 & 1 & 0 & -b_{4} & 1
\end{array}\right)
$$

up to the action of $\operatorname{GL}(4, \mathbb{Z})$ and $(\mathbb{Z} / 2)^{7}$, we only have to consider the two cases $\left(a_{2}, b_{4}\right)=(1,1)$ and $\left(a_{2}, b_{4}\right)=(1,-1)$.

- If $\left(a_{2}, b_{4}\right)=(1,1)$, we obtain $b_{1}=0,2$ from (7) and $c_{1}=b_{1} \pm 1$ from (6). Then $\lambda$ is of the form

$$
\lambda=\left(\begin{array}{cccc|ccc}
1 & 0 & 0 & 0 & 1 & b_{1} & b_{1} \pm 1 \\
0 & 1 & 0 & 0 & 1 & 1 & 1 \\
0 & 0 & 1 & 0 & 1 & 1 & 0 \\
0 & 0 & 0 & 1 & 0 & 1 & 1
\end{array}\right) \quad\left(b_{1}=0,2\right),
$$

and such $\lambda$ satisfies the nonsingularity condition. Thus we obtain the characteristic matrices $\lambda_{2}, \lambda_{9}, \lambda_{11}$ and $\lambda_{18}$.

- If $\left(a_{2}, b_{4}\right)=(1,-1)$, we obtain $b_{1}=0,-2$ from (7) and $c_{1}=-b_{1} \pm 1$ from (6). Moreover, $b_{1}+2 c_{1}-1= \pm 1$ from (11), so $c_{1}=1$. Then $\lambda$ is of the form

$$
\lambda=\left(\begin{array}{rrrr|rrr}
1 & 0 & 0 & 0 & 1 & b_{1} & 1 \\
0 & 1 & 0 & 0 & 1 & -1 & 1 \\
0 & 0 & 1 & 0 & 1 & 1 & 0 \\
0 & 0 & 0 & 1 & 0 & -1 & 1
\end{array}\right) \quad\left(b_{1}=0,-2\right),
$$

and such $\lambda$ satisfies the nonsingularity condition. When $b_{1}=0, \lambda$ coincides with $\lambda_{1}$ up to the action of $\operatorname{GL}(4, \mathbb{Z})$ and $(\mathbb{Z} / 2)^{7}$. Similarly, when $b_{1}=-2, \lambda$ coincides with $\lambda_{10}$. 
(i-B) If $\left(b_{2}-b_{4}\right) c_{1}=0$ and $a_{4}\left(b_{1}-1\right)= \pm 2$, we obtain $b_{2}=b_{4}$ and $a_{4}= \pm 2$, $b_{1}=1 \pm 1$. As in (i-A) above, we only have to consider two cases, $\left(a_{4}, b_{1}\right)=(2,0)$ and $\left(a_{4}, b_{1}\right)=(2,2)$.

- If $\left(a_{4}, b_{1}\right)=(2,0)$, we obtain $b_{4}=2 \pm 1$ from (3) and $b_{4}= \pm 1$ from (5), so $b_{4}=1$. Then we have $c_{1}= \pm 1$ from (6) and $a_{2}=2 \pm 1$ from (10). Moreover, $\left(a_{2}-1\right) c_{1}=1 \pm 1$ from (11). Then $\lambda$ is of the form

$$
\lambda=\left(\begin{array}{cccc|ccc}
1 & 0 & 0 & 0 & 1 & 0 & c_{1} \\
0 & 1 & 0 & 0 & a_{2} & 1 & 1 \\
0 & 0 & 1 & 0 & 1 & 1 & 0 \\
0 & 0 & 0 & 1 & 2 & 1 & 1
\end{array}\right),
$$

where $\left(a_{2}, c_{1}\right)$ is $(1, \pm 1)$ or $(3,1)$, and such $\lambda$ satisfies the nonsingularity condition. Thus we obtain the characteristic matrices $\lambda_{3}, \lambda_{12}$ and $\lambda_{16}$.

- If $\left(a_{4}, b_{1}\right)=(2,2)$, we obtain $b_{4}=2 \pm 1$ from (3) and $b_{4}=4 \pm 1$ from (5), so $b_{4}=3$. Then we have $2-3 c_{1}= \pm 1$ from (6), so $c_{1}=1$. Similarly, we obtain $a_{2}=1$ from (7). Then

$$
\lambda=\left(\begin{array}{llll|lll}
1 & 0 & 0 & 0 & 1 & 2 & 1 \\
0 & 1 & 0 & 0 & 1 & 3 & 1 \\
0 & 0 & 1 & 0 & 1 & 1 & 0 \\
0 & 0 & 0 & 1 & 2 & 3 & 1
\end{array}\right)
$$

satisfies the nonsingularity condition. Thus we obtain the characteristic matrix $\lambda_{4}$.

(i-C) If $\left(b_{2}-b_{4}\right) c_{1}= \pm 2$ and $a_{4}\left(b_{1}-1\right)=0$, we obtain $b_{2}-b_{4}= \pm 2, c_{1}= \pm 1$ and $a_{4}=0$. Similarly as before, we only have to consider the case $b_{2}-b_{4}=2$. From (3), $b_{4}= \pm 1$.

- If $b_{4}=1$, then $b_{2}=3$. We have $b_{1}= \pm 1 \pm 1$ from (6) and $b_{1}= \pm 1 \pm 3$ from (8), so $b_{1}= \pm 2$. Then we have $a_{2} b_{1}= \pm 2 a_{2}=3 \pm 1$ from (7) and $a_{2}=2 \pm 1$ from (10), so $a_{2}=1$ and $b_{1}=2$. Moreover, $c_{1}=1$ from (8). Then

$$
\lambda=\left(\begin{array}{llll|lll}
1 & 0 & 0 & 0 & 1 & 2 & 1 \\
0 & 1 & 0 & 0 & 1 & 3 & 1 \\
0 & 0 & 1 & 0 & 1 & 1 & 0 \\
0 & 0 & 0 & 1 & 0 & 1 & 1
\end{array}\right)
$$

satisfies the nonsingularity condition. Thus we obtain the characteristic matrix $\lambda_{6}$. 
- If $b_{4}=-1$, then $b_{2}=1$. We have $b_{1}+c_{1}= \pm 1$ from (6) and $b_{1}-c_{1}= \pm 1$ from (8), so $b_{1}=0$. We obtain $a_{2}=2 \pm 1$ from (10) and $-1+c_{1}\left(a_{2}-1\right)= \pm 1$ from (11). Then

$$
\lambda=\left(\begin{array}{rrrr|rrr}
1 & 0 & 0 & 0 & 1 & 0 & c_{1} \\
0 & 1 & 0 & 0 & a_{2} & 1 & 1 \\
0 & 0 & 1 & 0 & 1 & 1 & 0 \\
0 & 0 & 0 & 1 & 0 & -1 & 1
\end{array}\right)
$$

satisfies the nonsingularity condition, where $\left(a_{2}, c_{1}\right)=(1, \pm 1),(3,1)$. Thus we obtain the characteristic matrices $\lambda_{5}, \lambda_{13}$ and $\lambda_{15}$.

(i-D) If $\left(b_{2}-b_{4}\right) c_{1}= \pm 2$ and $a_{4}\left(b_{1}-1\right)= \pm 2$, we obtain $b_{2}-b_{4}= \pm 2, c_{1}= \pm 1$ and $a_{4}= \pm 2, b_{1}=1 \pm 1$. Similarly to the previous cases, we only have to consider one case, namely $a_{4}=2$.

- If $b_{1}=0$, then $b_{4}= \pm 1$ from (5) and $b_{2}= \pm 1$ from (7). In addition, $b_{4}=2 \pm 1$ from (3), so $b_{4}=1$ and $b_{2}=-1$. We obtain $a_{2}= \pm 1$ from (10) and $c_{1}\left(a_{2}+1\right)=1 \pm 1$ from (11). Then

$$
\lambda=\left(\begin{array}{rrrr|rrr}
1 & 0 & 0 & 0 & 1 & 0 & c_{1} \\
0 & 1 & 0 & 0 & a_{2} & -1 & 1 \\
0 & 0 & 1 & 0 & 1 & 1 & 0 \\
0 & 0 & 0 & 1 & 2 & 1 & 1
\end{array}\right)
$$

satisfies the nonsingularity condition, where $\left(a_{2}, c_{1}\right)=(-1, \pm 1),(1,1)$. Thus we obtain the characteristic matrices $\lambda_{7}, \lambda_{14}$ and $\lambda_{17}$.

- If $b_{1}=2$, then we have $b_{4}=2 \pm 1$ from (3) and $b_{4}=4 \pm 1$ from (5), so $b_{4}=3$. From (8), $b_{2}= \pm(-2 \pm 1)$, and since $b_{2}-b_{4}= \pm 2$, we see that $b_{2}=1$. Then we obtain $a_{2}=1$ from (7). Similarly, we obtain $c_{1}=1$ from (6). Then

$$
\lambda=\left(\begin{array}{llll|lll}
1 & 0 & 0 & 0 & 1 & 2 & 1 \\
0 & 1 & 0 & 0 & 1 & 1 & 1 \\
0 & 0 & 1 & 0 & 1 & 1 & 0 \\
0 & 0 & 0 & 1 & 2 & 3 & 1
\end{array}\right)
$$

satisfies the nonsingularity condition. Thus we obtain the characteristic matrix $\lambda_{8}$.

(ii) Next, we consider the case $|1,4,5,7|_{\lambda}=a_{2} c_{3}-1=1$, namely $\left(a_{2}, c_{3}\right)=$ $(1,2),(-1,-2)$. Since $\lambda$ coincides with

$$
\left(\begin{array}{rrrr|rrr}
1 & 0 & 0 & 0 & 1 & b_{1} & -c_{1} \\
0 & 1 & 0 & 0 & -a_{2} & -b_{2} & 1 \\
0 & 0 & 1 & 0 & 1 & b_{3} & -c_{3} \\
0 & 0 & 0 & 1 & -a_{4} & -b_{4} & 1
\end{array}\right)
$$


up to the action of $\operatorname{GL}(4, \mathbb{Z})$ and $(\mathbb{Z} / 2)^{7}$, we can assume $\left(a_{2}, c_{3}\right)=(1,2)$. Moreover, since we assume $b_{3}>0$, we obtain $b_{4}>0$ from (4). In the same way as in case (i), we obtain $\left(b_{2}-b_{4}\right) c_{1}=0, \pm 2$ and $a_{4}\left(b_{1}-b_{3}\right)=0, \pm 2$.

(ii-A) If $\left(b_{2}-b_{4}\right) c_{1}=0$ and $a_{4}\left(b_{1}-b_{3}\right)=0$, we obtain $b_{2}=b_{4}$ and $a_{4}=0$. From (3), $b_{4}=1$. Then

$$
\lambda=\left(\begin{array}{cccc|ccc}
1 & 0 & 0 & 0 & 1 & b_{1} & c_{1} \\
0 & 1 & 0 & 0 & 1 & 1 & 1 \\
0 & 0 & 1 & 0 & 1 & b_{3} & 2 \\
0 & 0 & 0 & 1 & 0 & 1 & 1
\end{array}\right) .
$$

We have $b_{3}=2 \pm 1$ from (4), $b_{1}=1 \pm 1$ from (7) and $c_{1}=b_{1} \pm 1$ from (6).

- If $b_{3}=1$, for any pair $\left(b_{1}, c_{1}\right)$ such that $b_{1}=1 \pm 1$ and $c_{1}=b_{1} \pm 1, \lambda$ satisfies the nonsingularity condition. Thus we obtain the characteristic matrices $\lambda_{20}, \lambda_{21}, \lambda_{26}$ and $\lambda_{28}$.

- If $b_{3}=3$, we have $2 c_{1}-b_{1}=1 \pm 1$ from (11), so $c_{1}=1$. Then

$$
\lambda=\left(\begin{array}{llll|lcl}
1 & 0 & 0 & 0 & 1 & 1 \pm 1 & 1 \\
0 & 1 & 0 & 0 & 1 & 1 & 1 \\
0 & 0 & 1 & 0 & 1 & 3 & 2 \\
0 & 0 & 0 & 1 & 0 & 1 & 1
\end{array}\right)
$$

satisfies the nonsingularity condition. Thus we get the characteristic matrices $\lambda_{19}$ and $\lambda_{22}$.

(ii-B) If $\left(b_{2}-b_{4}\right) c_{1}=0$ and $a_{4}\left(b_{1}-b_{3}\right)= \pm 2$, we obtain $b_{2}=b_{4}$ and $a_{4}= \pm 2$, $b_{1}=b_{3} \pm 1$. Since $1-a_{4}= \pm 1$ from (10), we obtain $a_{4}=2$. From (3) and (4), $3\left(b_{3}-b_{4}\right)= \pm 1 \pm 1$, so we get $b_{3}=b_{4}=1$. (Notice that $b_{4}>0$.) Moreover, we obtain $b_{1}=0$ from (5) and $c_{1}= \pm 1$ from (6). Then

$$
\lambda=\left(\begin{array}{llll|lcc}
1 & 0 & 0 & 0 & 1 & 0 & \pm 1 \\
0 & 1 & 0 & 0 & 1 & 1 & 1 \\
0 & 0 & 1 & 0 & 1 & 1 & 2 \\
0 & 0 & 0 & 1 & 2 & 1 & 1
\end{array}\right)
$$

satisfies the nonsingularity condition. Thus we get the characteristic matrices $\lambda_{25}$ and $\lambda_{27}$.

(ii-C) If $\left(b_{2}-b_{4}\right) c_{1}= \pm 2$ and $a_{4}\left(b_{1}-b_{3}\right)=0$, we obtain $b_{2}-b_{4}= \pm 2, c_{1}= \pm 1$ and $a_{4}=0$. Then $b_{4}=1$ from (3). 
- If $b_{2}-b_{4}=2$, then $b_{2}=3$. We have $b_{1}= \pm 1 \pm 1$ from (6) and $b_{1}=3 \pm 1$ from (7), so $b_{1}=2$. Then $4-4 c_{1}= \pm 1 \pm 1$ from (6) and (8), so $c_{1}=1$. Moreover, $b_{3}=3$ from (4) and (10). Then

$$
\lambda=\left(\begin{array}{llll|lll}
1 & 0 & 0 & 0 & 1 & 2 & 1 \\
0 & 1 & 0 & 0 & 1 & 3 & 1 \\
0 & 0 & 1 & 0 & 1 & 3 & 2 \\
0 & 0 & 0 & 1 & 0 & 1 & 1
\end{array}\right)
$$

satisfies the nonsingularity condition, so we obtain the characteristic matrix $\lambda_{24}$.

- If $b_{2}-b_{4}=-2$, then $b_{2}=-1$. Then $2 b_{1}= \pm 1 \pm 1$ from (6) and (8), so $b_{1}=0$. From (4) and (10), we obtain $b_{3}=1$. Moreover, $2 c_{1}=3 \pm 1$ from (11), so $c_{1}=1$. Then

$$
\lambda=\left(\begin{array}{rrrr|rrr}
1 & 0 & 0 & 0 & 1 & 0 & 1 \\
0 & 1 & 0 & 0 & 1 & -1 & 1 \\
0 & 0 & 1 & 0 & 1 & 1 & 2 \\
0 & 0 & 0 & 1 & 0 & 1 & 1
\end{array}\right)
$$

satisfies the nonsingularity condition, so we obtain the characteristic matrix $\lambda_{23}$.

(ii-D) If $\left(b_{2}-b_{4}\right) c_{1}= \pm 2$ and $a_{4}\left(b_{1}-b_{3}\right)= \pm 2$, we obtain $b_{2}-b_{4}= \pm 2, c_{1}= \pm 1$ and $a_{4}= \pm 2, b_{1}=b_{3} \pm 1$. If we assume $a_{4}=-2$, then $b_{4}+2 b_{3} \geq 3$, but this contradicts (3). Thus $a_{4}=2$. From (3) and (4), $3\left(b_{3}-b_{4}\right)= \pm 1 \pm 1$, so $b_{3}=b_{4}=1$. We obtain $b_{1}=0$ from (5), and hence $b_{2}=-1$ from (7) and $b_{2}-b_{4}= \pm 2$. Then $|1,5,6,7|_{\lambda}=-5$, but this contradicts (10).

Thus we obtain all characteristic matrices on $C^{4}(7)^{*}$ up to the action of $\operatorname{GL}(4, \mathbb{Z})$ and $(\mathbb{Z} / 2)^{7}$.

Next, we consider the action of $\sigma$ on $p^{-1}(\bar{\lambda})$. By a direct calculation, we see that the action is illustrated as follows:

$$
\begin{aligned}
& \lambda_{1} \stackrel{\sigma}{\longrightarrow} \lambda_{9} \stackrel{\sigma}{\longrightarrow} \lambda_{5} \stackrel{\sigma}{\longrightarrow} \lambda_{21} \stackrel{\sigma}{\longrightarrow} \lambda_{3} \stackrel{\sigma}{\longrightarrow} \lambda_{2} \stackrel{\sigma}{\longrightarrow} \lambda_{11} \stackrel{\sigma}{\longrightarrow} \lambda_{1} \\
& \lambda_{4} \stackrel{\sigma}{\longrightarrow} \lambda_{18} \stackrel{\sigma}{\longrightarrow} \lambda_{13} \stackrel{\sigma}{\longrightarrow} \lambda_{19} \stackrel{\sigma}{\longrightarrow} \lambda_{17} \stackrel{\sigma}{\longrightarrow} \lambda_{14} \stackrel{\sigma}{\longrightarrow} \lambda_{27} \stackrel{\sigma}{\longrightarrow} \lambda_{4} \\
& \lambda_{6} \stackrel{\sigma}{\longrightarrow} \lambda_{26} \stackrel{\sigma}{\longrightarrow} \lambda_{7} \stackrel{\sigma}{\longrightarrow} \lambda_{20} \stackrel{\sigma}{\longrightarrow} \lambda_{14} \stackrel{\sigma}{\longrightarrow} \lambda_{22} \stackrel{\sigma}{\longrightarrow} \lambda_{16} \stackrel{\sigma}{\longrightarrow} \lambda_{6} \\
& \lambda_{8} \stackrel{\sigma}{\longrightarrow} \lambda_{28} \stackrel{\sigma}{\longrightarrow} \lambda_{12} \stackrel{\sigma}{\longrightarrow} \lambda_{10} \stackrel{\sigma}{\longrightarrow} \lambda_{15} \stackrel{\sigma}{\longrightarrow} \lambda_{23} \stackrel{\sigma}{\longrightarrow} \lambda_{25} \stackrel{\sigma}{\longrightarrow} \lambda_{8}
\end{aligned}
$$

Then we obtain the following proposition.

Proposition 7.3 Over $C^{4}(7)^{*}$, there are exactly four quasitoric manifolds $M\left(\lambda_{9}\right)$, $M\left(\lambda_{17}\right), M\left(\lambda_{16}\right)$ and $M\left(\lambda_{15}\right)$ up to weakly equivariant homeomorphism. 
Next, putting $A:=H^{*}\left(\lambda_{9}\right), B:=H^{*}\left(\lambda_{17}\right), C:=H^{*}\left(\lambda_{16}\right), D:=H^{*}\left(\lambda_{15}\right)$, let us show that $A, B, C, D$ are not isomorphic to each other as graded rings. $\operatorname{Miss}\left(K_{C^{4}(7)^{*}}\right)$ consists of $\{1,3,5\},\{1,3,6\},\{1,4,6\},\{2,4,6\},\{2,4,7\},\{2,5,7\}$ and $\{3,5,7\}$, so $\mathcal{I}_{C^{4}(7)^{*}}$ in Theorem 2.21 is generated by $v_{1} v_{3} v_{5}, v_{1} v_{3} v_{6}, v_{1} v_{4} v_{6}, v_{2} v_{4} v_{6}, v_{2} v_{4} v_{7}$, $v_{2} v_{5} v_{7}$ and $v_{3} v_{5} v_{7}$. With the notation of Theorem 2.21, we put $X:=v_{5}, Y:=v_{6}$, $Z:=v_{7}$. We can take $[M]:=v_{1} v_{5} v_{6} v_{7}$ as the fundamental cohomology class. Indeed, each $v_{i}$ is the Poincaré dual of $\pi^{-1}\left(F_{i}\right)$, and $\pi^{-1}\left(F_{1}\right), \pi^{-1}\left(F_{5}\right), \pi^{-1}\left(F_{6}\right)$ and $\pi^{-1}\left(F_{7}\right)$ intersect transversally at the point $\pi^{-1}(v)$, where $v$ denotes the vertex $F_{1} \cap F_{5} \cap F_{6} \cap F_{7}$. Table 1 shows the coefficients of $[M]$ in each monomial.

\begin{tabular}{rrrrr} 
& $A$ & $B$ & $C$ & $D$ \\
\hline$X^{4}$ & 0 & 0 & 0 & 0 \\
$Y^{4}$ & 0 & -4 & 6 & -2 \\
$Z^{4}$ & -2 & 0 & -2 & 0 \\
$X^{3} Y$ & 0 & 0 & 0 & 0 \\
$X^{2} Y^{2}$ & 0 & 0 & 0 & 0 \\
$X Y^{3}$ & 1 & 1 & -1 & 1 \\
$Y^{3} Z$ & 0 & 2 & 0 & 2 \\
$Y^{2} Z^{2}$ & 0 & 0 & 0 & 0 \\
$Y Z^{3}$ & 1 & 1 & 1 & 1 \\
$Z^{3} X$ & 0 & 0 & 0 & 0 \\
$Z^{2} X^{2}$ & 0 & 0 & 0 & 0 \\
$Z X^{3}$ & -1 & -1 & -1 & -1 \\
$X^{2} Y Z$ & 1 & 1 & 1 & 1 \\
$X Y^{2} Z$ & -1 & -1 & -1 & -1 \\
$X Y Z^{2}$ & 0 & 0 & 0 & 0
\end{tabular}

Table 1

Lemma 7.4 $B$ is not isomorphic to $A, C$ or $D$.

Proof Let us denote the generators $X, Y, Z$ of $A$ by $X_{1}, Y_{1}, Z_{1}$. Similarly, denote the generators of $B$ by $X_{2}, Y_{2}, Z_{2}$. If we assume that there exists a graded ring isomorphism $\phi: A \rightarrow B$, then $\pm 2[M]=\phi\left(\left(Z_{1}\right)^{4}\right)=\phi\left(Z_{1}\right)^{4}=\left(a X_{2}+b Y_{2}+c Z_{2}\right)^{4}$ for some integers $a, b, c$. However, since $\left(X_{2}\right)^{4}=\left(Z_{2}\right)^{4}=\left(X_{2} Y_{2}\right)^{2}=\left(Y_{2} Z_{2}\right)^{2}=$ $\left(Z_{2} X_{2}\right)^{2}=0$ and $\left(Y_{2}\right)^{4} \equiv 0 \bmod 4$, we see that $\left(a X_{2}+b Y_{2}+c Z_{2}\right)^{4} \equiv 0 \bmod 4$. This is a contradiction. We can prove the lemma similarly for $C$ and $D$. 
Lemma 7.5 $A$ and $C$ are not isomorphic to $D$.

Proof It suffices to show that there exists no graded ring isomorphism from $A /(3)$ or $C /(3)$ to $D /(3)$. Assume there exists a graded ring isomorphism $\phi: A /(3) \rightarrow D /(3)$, and take vectors $v_{i}=\left(a_{i}, b_{i}, c_{i}\right) \in(\mathbb{Z} / 3)^{3}$ for $i=1,2,3$ such that

$$
\left(\begin{array}{l}
\phi(X) \\
\phi(Y) \\
\phi(Z)
\end{array}\right)=\left(\begin{array}{lll}
a_{1} & b_{1} & c_{1} \\
a_{2} & b_{2} & c_{2} \\
a_{3} & b_{3} & c_{3}
\end{array}\right)\left(\begin{array}{l}
X \\
Y \\
Z
\end{array}\right) .
$$

Note that for any $x \in \mathbb{Z} / 3$ we have $x^{3}=x$. From $\phi(X)^{4}=0$, we obtain the equation $2 a_{1} c_{1}+a_{1} b_{1}+b_{1}^{2}=0$. Similarly, from $\phi(Y)^{4}=0$, we obtain $2 a_{2} c_{2}+a_{2} b_{2}+b_{2}^{2}=0$. From a direct calculation, we see that

$$
v_{i}= \pm(0,0,1), \pm(1,0,0), \pm(1,1,2), \pm(1,2,0)
$$

for $i=1,2$.

- If $v_{1}= \pm(0,0,1)$, from $\phi\left(X^{3} Y\right)=0$ and the list above, we have $v_{2}= \pm(1,0,0)$. Then, from $\phi\left(Z^{3} X\right)=\phi\left(Y^{3} Z\right)=0$, we obtain $v_{3}= \pm(1,2,0)$, but this is a contradiction since $0 \neq \phi\left(Z^{4}\right)= \pm(X+2 Y)^{4}=0$.

- If $v_{1}= \pm(1,0,0)$, from $\phi\left(X^{3} Y\right)=0$ and the list above, we have $v_{2}= \pm(1,2,0)$. Then, from $\phi\left(Z^{3} X\right)=\phi\left(Y^{3} Z\right)=0$, we obtain $v_{3}= \pm(0,0,1)$, but this is a contradiction since $0 \neq \phi\left(Z^{4}\right)= \pm Z^{4}=0$.

- If $v_{1}= \pm(1,1,2)$, from $\phi\left(X^{3} Y\right)=0$ and the list above, we have $v_{2}= \pm(1,1,2)$, but this contradicts the linear independence of $v_{1}$ and $v_{2}$.

- If $v_{1}= \pm(1,2,0)$, from $\phi\left(X^{3} Y\right)=0$ and the list above, we have $v_{2}= \pm(0,0,1)$. Then, from $\phi\left(Z^{3} X\right)=\phi\left(Y^{3} Z\right)=0$, we obtain $v_{3}= \pm(1,0,0)$, but this is a contradiction since $0 \neq \phi\left(Z^{4}\right)= \pm X^{4}=0$.

This proof also works when we consider $C$ instead of $A$.

Lemma 7.6 $A$ and $C$ are not isomorphic as graded rings.

Proof We prove that there exists no graded ring isomorphism from $A /(3)$ to $C /(3)$. Let us assume that there exists a graded ring isomorphism $\phi: A /(3) \rightarrow C /(3)$. Using the same notation as in the proof of the previous lemma, from $\phi\left(X^{4}\right)=\phi\left(Y^{4}\right)=0$ we obtain the equations $c_{i}^{2}+2 a_{i} b_{i}+b_{i} c_{i}+2 a_{i} c_{i}=0$ for $i=1,2$. Then we see that $v_{i}= \pm(0,1,0), \pm(0,1,2), \pm(1,0,0), \pm(1,0,1), \pm(1,1,1), \pm(1,1,2), \pm(1,2,1)$ for $i=1,2$. 
- If $v_{1}= \pm(0,1,0)$, from $\phi\left(X^{3} Y\right)=0$ and the list above, we have $v_{2}= \pm(0,1,2)$. Then, from $\phi\left(Z^{3} X\right)=\phi\left(Y^{3} Z\right)=0$, we obtain $v_{3}= \pm(1,2,0)$, but this is a contradiction since $0=\phi\left(Z^{2} X^{2}\right) \neq 0$. This proof also works in the case that $v_{1}= \pm(1,1,1)$.

- If $v_{1}= \pm(0,1,2)$, from $\phi\left(X^{3} Y\right)=0$ and the list above, we have $v_{2}= \pm(1,1,1)$. Then $0=\phi\left(X^{2} Y^{2}\right) \neq 0$, which is a contradiction. This proof also works when $v_{1}= \pm(1,0,1), \pm(1,1,2)$.

- If $v_{1}= \pm(1,0,0)$, from $\phi\left(X^{3} Y\right)=0$ and the list above, we have $v_{2}=(0, b, 0)$ for $b= \pm 1$. Then, from $\phi\left(Z^{3} X\right)=\phi\left(Y^{3} Z\right)=0$, we obtain $v_{3}= \pm(0,0, c)$ for $c= \pm 1$. Besides, from $Z^{4}=X Y^{3}=Y Z^{3}=2 Z X^{3}$ in $A /(3)$, we have $1=2 b=b c=c$. However, this equation has no root.

- If $v_{1}= \pm(1,2,1)$, from $\phi\left(X^{3} Y\right)=0$ and the list above, we have $v_{2}= \pm(1,2,1)$. This contradicts the linear independence of $v_{1}$ and $v_{2}$.

Hence we see that there exists no isomorphism $A /(3) \rightarrow C /(3)$.

Thus we have completed the topological classification of quasitoric manifolds over $C^{4}(7)^{*}$.

Theorem 7.7 Any quasitoric manifold over $C^{4}(7)^{*}$ is homeomorphic to $M\left(\lambda_{9}\right)$, $M\left(\lambda_{17}\right), M\left(\lambda_{16}\right)$ or $M\left(\lambda_{15}\right)$, and the cohomology rings of those manifolds are not isomorphic.

\section{Classification for $C^{5}(8)^{*}$}

In Section 6, we showed that $\mathbb{R}_{C^{5}(8)^{*}}$ has exactly two elements and they are represented by the real characteristic matrices

$$
\left(\begin{array}{lllll|lll}
1 & 0 & 0 & 0 & 0 & 0 & 0 & 1 \\
0 & 1 & 0 & 0 & 0 & 1 & 0 & 1 \\
0 & 0 & 1 & 0 & 0 & 1 & 1 & 1 \\
0 & 0 & 0 & 1 & 0 & 1 & 1 & 0 \\
0 & 0 & 0 & 0 & 1 & 0 & 1 & 1
\end{array}\right), \quad\left(\begin{array}{lllll|lll}
1 & 0 & 0 & 0 & 0 & 0 & 0 & 1 \\
0 & 1 & 0 & 0 & 0 & 1 & 1 & 0 \\
0 & 0 & 1 & 0 & 0 & 0 & 1 & 1 \\
0 & 0 & 0 & 1 & 0 & 1 & 1 & 1 \\
0 & 0 & 0 & 0 & 1 & 1 & 0 & 1
\end{array}\right) .
$$

As in the previous section, let us denote these two matrices by $\bar{\lambda}, \bar{\lambda}^{\prime}$ and the map $\mathcal{X}_{C^{5}(8)^{*}} \rightarrow \mathbb{R}_{C^{5}(8)^{*}}$ by $p$. It is shown in the same way as in Lemma 5.3 that Aut $\left(K_{C^{5}(8)^{*}}\right)$ is generated by

$$
\sigma=\left(\begin{array}{llllllll}
1 & 2 & 3 & 4 & 5 & 6 & 7 & 8 \\
8 & 2 & 3 & 4 & 5 & 6 & 7 & 1
\end{array}\right) \text { and } \quad \tau=\left(\begin{array}{llllllll}
1 & 2 & 3 & 4 & 5 & 6 & 7 & 8 \\
8 & 7 & 6 & 5 & 4 & 3 & 2 & 1
\end{array}\right)
$$


They act on $\mathbb{R}_{C^{5}(8)^{*}}$ by $\sigma(\bar{\lambda})=\bar{\lambda}, \sigma\left(\bar{\lambda}^{\prime}\right)=\bar{\lambda}^{\prime}$ and $\tau(\bar{\lambda})=\bar{\lambda}^{\prime}$. Then we obtain the following proposition.

Proposition 8.1 There exists only one small cover over $C^{5}(8)^{*}$ up to weakly equivariant homeomorphism.

We also see that $p^{-1}(\bar{\lambda}) /\langle\sigma\rangle$ corresponds to $\mathcal{X}_{C^{5}(8)^{*}} /$ Aut $\left(K_{C^{5}(8)^{*}}\right)$. Any element in $p^{-1}(\bar{\lambda})$ is represented by a characteristic matrix of the form

$$
\left(\lambda_{k} ; a, b\right):=\left(\begin{array}{c|ccccccc}
1 & 0 & 0 & 0 & 0 & a & b & 1 \\
\hline 0 & & & & & & \\
0 & & & & & & \\
0 & & & \lambda_{k} & & \\
0 & & & & & &
\end{array}\right)=\left(\begin{array}{ccccc|ccc}
1 & 0 & 0 & 0 & 0 & a & b & 1 \\
0 & 1 & 0 & 0 & 0 & 1 & b_{1} & c_{1} \\
0 & 0 & 1 & 0 & 0 & a_{2} & b_{2} & 1 \\
0 & 0 & 0 & 1 & 0 & 1 & b_{3} & c_{3} \\
0 & 0 & 0 & 0 & 1 & a_{4} & b_{4} & 1
\end{array}\right),
$$

where $a, b$ are even integers and $\lambda_{k}(k=1,2, \ldots, 28)$ are the characteristic matrices on $C^{4}(7)^{*}$ given in Proposition 7.2. Notice that $\left(\lambda_{k} ; a, b\right)$ satisfies the equality $\left|1, i_{1}, i_{2}, i_{3}, i_{4}\right|= \pm 1$ for each $\left\{1, i_{1}, i_{2}, i_{3}, i_{4}\right\} \in K_{C^{5}(8)^{*}}$. Therefore, $\left(\lambda_{k} ; a, b\right)$ is a characteristic matrix on $C^{5}(8)^{*}$ if and only if the following equalities hold: $|2,3,5,6,8|=a c_{3}-1= \pm 1,|3,4,5,6,8|=a c_{1}-1= \pm 1,|2,3,6,7,8|= \pm 1$, $|3,4,6,7,8|= \pm 1,|4,5,6,7,8|= \pm 1$. Solving these equations, we obtain sixty-four characteristic matrices up to the action of $\operatorname{GL}(5, \mathbb{Z})$ and $(\mathbb{Z} / 2)^{8}$, and the action of $\sigma$ is illustrated in the figure on page 1429.

Hence we obtain the following proposition.

Proposition 8.2 There are exactly forty-six quasitoric manifolds over $C^{5}(8)^{*}$ up to weakly equivariant homeomorphism.

We will show that the cohomology rings of these quasitoric manifolds are not isomorphic. Notice that $\operatorname{Miss}\left(K_{C^{5}(8)^{*}}\right)$ consists of $\{2,4,6\},\{2,4,7\},\{2,5,7\},\{3,5,7\}$, $\{1,3,5,8\},\{1,3,6,8\}$ and $\{1,4,6,8\}$, so $\mathcal{I}_{C^{5}(8) *}$ in Theorem 2.21 is generated by $v_{2} v_{4} v_{6}, v_{2} v_{4} v_{7}, v_{2} v_{5} v_{7}, v_{3} v_{5} v_{7}, v_{1} v_{3} v_{5} v_{8}, v_{1} v_{3} v_{6} v_{8}$ and $v_{1} v_{4} v_{6} v_{8}$.

For a characteristic matrix $\xi=\left(\lambda_{k} ; a, b\right)=\left(\lambda_{i, j}\right)$ on $C^{5}(8)^{*}$, let us denote $v_{i_{1}}^{\prime} \cdots v_{i_{k}}^{\prime}$ in Theorem 3.4 by $\left[i_{1}, \ldots, i_{k}\right]_{\xi}$ or simply $\left[i_{1}, \ldots, i_{k}\right]$. Put $X:=v_{6}, Y:=v_{7}, Z:=v_{8}$. Then we obtain $H^{*}(\xi) \cong \mathbb{Z}[X, Y, Z] /\left(\mathcal{I}_{\xi}^{6}+\mathcal{I}_{\xi}^{8}\right)$, where $\mathcal{I}_{\xi}^{6}$ and $\mathcal{I}_{\xi}^{8}$ are the ideals

$$
\begin{aligned}
\mathcal{I}_{\xi}^{6} & =([2,4,6],[2,4,7],[2,5,7],[3,5,7]), \\
\mathcal{I}_{\xi}^{8} & =([1,3,5,8],[1,3,6,8],[1,4,6,8]) .
\end{aligned}
$$

Note that $\mathcal{I}_{\xi}^{6}$ is determined by $\lambda_{k}$ (namely, independent of $a$ and $b$ ). Hence we write $\mathcal{I}_{k}^{6}=\mathcal{I}_{\xi}^{6}$. 

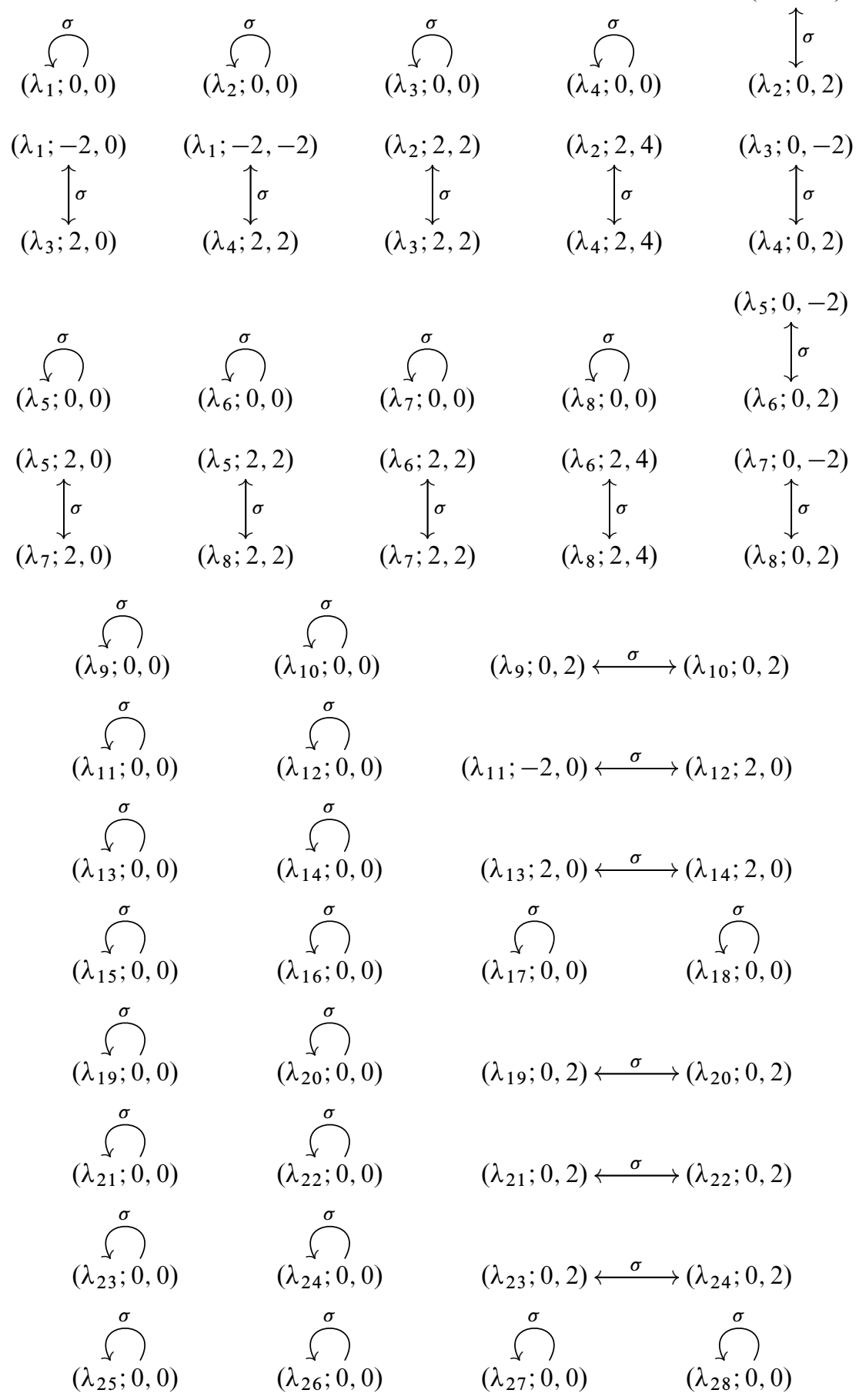
Definition 8.3 For $k, k^{\prime}=1,2, \ldots, 28$, we denote by $\mathcal{A}_{k, k^{\prime}}$ the set of all graded ring automorphisms of $\mathbb{Z}[X, Y, Z]$ that map $\mathcal{I}_{k}^{6}$ into $\mathcal{I}_{k^{\prime}}^{6}$.

Now we briefly explain the procedure for classifying quasitoric manifolds over $C^{5}(8)^{*}$. First, we list all elements of $\mathcal{A}_{k, k^{\prime}}$ for any $k, k^{\prime}$. Then we only have to verify that those automorphisms do not map

$$
\mathcal{I}_{\left(\lambda_{k} ; a, b\right)}^{8} \rightarrow \mathcal{I}_{k^{\prime}}^{6}+\mathcal{I}_{\left(\lambda_{k^{\prime}} ; a^{\prime}, b^{\prime}\right)}^{8}
$$

for any combination of even integers $a, b, a^{\prime}, b^{\prime}$ such that $\left(\lambda_{k} ; a, b\right)$ and $\left(\lambda_{k^{\prime}} ; a^{\prime}, b^{\prime}\right)$ are characteristic matrices on $C^{5}(8)^{*}$. Since each $\mathcal{A}_{k, k^{\prime}}$ proves to have at most 8 elements, we can do this by means of a computer-assisted calculation.

Let us define the equivalence relation $\sim_{\sigma}$ on $\{1, \ldots, 28\}$ as follows: $k \sim_{\sigma} k^{\prime}$ if and only if $\sigma\left(\left(\lambda_{k} ; a, b\right)\right)=\left(\lambda_{k^{\prime}} ; a^{\prime}, b^{\prime}\right)$ for some $a, b, a^{\prime}, b^{\prime}$ such that $\left(\lambda_{k} ; a, b\right)$ and $\left(\lambda_{k^{\prime}} ; a^{\prime}, b^{\prime}\right)$ are characteristic matrices on $C^{5}(8)^{*}$. Then the equivalence classes for $\sim_{\sigma}$ are $\{1,2,3,4\},\{5,6,7,8\},\{9,10\},\{11,12\},\{13,14\},\{15\},\{16\},\{17\},\{18\}$, $\{19,20\},\{21,22\},\{23,24\},\{25\},\{26\},\{27\},\{28\}$.

Remark 8.4 Let $\lambda=\left(\lambda_{k} ; a, b\right)$ and $\lambda^{\prime}=\left(\lambda_{k^{\prime}} ; a^{\prime}, b^{\prime}\right)$ be characteristic matrices on $C^{5}(8)^{*}$ such that $\sigma(\lambda)=\lambda^{\prime}$. By Remark 2.22, there is a graded ring automorphism $\phi_{\left(\sigma ; \lambda, \lambda^{\prime}\right)}$ of $\mathbb{Z}[X, Y, Z]$ which maps $\mathcal{I}_{\lambda}$ into $\mathcal{I}_{\lambda^{\prime}}$, in particular, $\mathcal{I}_{k}^{6}$ into $\mathcal{I}_{k^{\prime}}^{6}$. Then we only have to determine $\mathcal{A}_{k, k^{\prime}}$ for $k, k^{\prime} \in K$, where

$$
K:=\{1,5,9,11,13,15,16,17,18,19,21,23,25,26,27,28\} .
$$

Hereafter we assume that a graded ring automorphism $\phi$ of $\mathbb{Z}[X, Y, Z]$ maps $\mathcal{I}_{\lambda}^{6}$ into $\mathcal{I}_{\lambda^{\prime}}^{6}$, where $\lambda=\left(\lambda_{k} ; a, b\right)$ and $\lambda^{\prime}=\left(\lambda_{k^{\prime}} ; a^{\prime}, b^{\prime}\right)$ are two characteristic matrices with $k, k^{\prime} \in K$. Write

$$
\lambda=\left(\begin{array}{ccccc|ccc}
1 & 0 & 0 & 0 & 0 & a & b & 1 \\
0 & 1 & 0 & 0 & 0 & 1 & p & q \\
0 & 0 & 1 & 0 & 0 & x & y & 1 \\
0 & 0 & 0 & 1 & 0 & 1 & s & t \\
0 & 0 & 0 & 0 & 1 & m & n & 1
\end{array}\right), \quad \lambda^{\prime}=\left(\begin{array}{ccccc|ccc}
1 & 0 & 0 & 0 & 0 & a^{\prime} & b^{\prime} & 1 \\
0 & 1 & 0 & 0 & 0 & 1 & p^{\prime} & q^{\prime} \\
0 & 0 & 1 & 0 & 0 & x^{\prime} & y^{\prime} & 1 \\
0 & 0 & 0 & 1 & 0 & 1 & s^{\prime} & t^{\prime} \\
0 & 0 & 0 & 0 & 1 & m^{\prime} & n^{\prime} & 1
\end{array}\right) .
$$

It is convenient to identify a homogeneous polynomial $f$ of degree 6 in $\mathbb{Z}[X, Y, Z]$ with the row vector $u$ defined through

$$
f=\left(X^{3}, Y^{3}, Z^{3}, X^{2} Y, X Y^{2}, Y^{2} Z, Y Z^{2}, Z^{2} X, Z X^{2}, X Y Z\right)^{t} u .
$$


Then we see that ${ }^{t}\left([2,4,6]_{\lambda},[2,4,7]_{\lambda},[2,5,7]_{\lambda},[3,5,7]_{\lambda}\right)$ is identified with the matrix

$$
\left(\begin{array}{cccccccccc}
1 & 0 & 0 & p+s & p s & 0 & 0 & q t & q+t & p t+q s \\
0 & p s & 0 & 1 & p+s & p t+q s & q t & 0 & 0 & t+q \\
0 & p n & 0 & m & p m+n & p+q n & q & 0 & 0 & 1+q m \\
0 & y n & 0 & x m & y m+x n & y+n & 1 & 0 & 0 & x+m
\end{array}\right),
$$

which is congruent with the following matrix modulo 2 :

$$
\left(\begin{array}{llllllllll}
1 & 0 & 0 & 1 & 0 & 0 & 0 & 0 & 1 & 1 \\
0 & 0 & 0 & 1 & 1 & 1 & 0 & 0 & 0 & 1 \\
0 & 0 & 0 & 0 & 1 & 1 & 1 & 0 & 0 & 1 \\
0 & 1 & 0 & 0 & 1 & 0 & 1 & 0 & 0 & 1
\end{array}\right)
$$

As in Section 5, we write

$$
\left(\begin{array}{l}
\phi(X) \\
\phi(Y) \\
\phi(Z)
\end{array}\right)=\left(\begin{array}{lll}
a_{1} & b_{1} & c_{1} \\
a_{2} & b_{2} & c_{2} \\
a_{3} & b_{3} & c_{3}
\end{array}\right)\left(\begin{array}{l}
X \\
Y \\
Z
\end{array}\right)
$$

and put $a:=\left(a_{1}, a_{2}, a_{3}\right), b:=\left(b_{1}, b_{2}, b_{3}\right), c:=\left(c_{1}, c_{2}, c_{3}\right)$. Then we may denote $\phi$ by the matrix $\left({ }^{t} a,{ }^{t} b,{ }^{t} c\right)$.

Lemma 8.5

$$
\phi \equiv\left(\begin{array}{lll}
1 & 0 & 0 \\
0 & 1 & 0 \\
0 & 0 & 1
\end{array}\right) \bmod 2
$$

Proof By row operations, we transform the matrix (13) into

$$
\left(\begin{array}{llllllllll}
1 & 0 & 0 & 0 & 0 & 0 & 1 & 0 & 1 & 1 \\
0 & 0 & 0 & 1 & 0 & 0 & 1 & 0 & 0 & 0 \\
0 & 0 & 0 & 0 & 1 & 1 & 1 & 0 & 0 & 1 \\
0 & 1 & 0 & 0 & 0 & 1 & 0 & 0 & 0 & 0
\end{array}\right)
$$

Since the coefficients of $Z^{3}$ in $\phi([2,4,6]), \phi([2,4,7]), \phi([2,5,7])$ and $\phi([3,5,7])$ are equal to zero (see the matrix (12)), we obtain

$$
\left(\begin{array}{c}
\left(c_{1}+p c_{2}+q c_{3}\right)\left(c_{1}+s c_{2}+t c_{3}\right) c_{1} \\
\left(c_{1}+p c_{2}+q c_{3}\right)\left(c_{1}+s c_{2}+t c_{3}\right) c_{2} \\
\left(c_{1}+p c_{2}+q c_{3}\right)\left(m c_{1}+n c_{2}+c_{3}\right) c_{2} \\
\left(x c_{1}+y c_{2}+c_{3}\right)\left(m c_{1}+n c_{2}+c_{3}\right) c_{2}
\end{array}\right)=0
$$

- If $c_{1} \equiv 1$ and $c_{2} \equiv 0 \bmod 2$, we have $c_{3} \equiv 1 \bmod 2$. Since the coefficient of $Z^{2} X$ in $\phi([2,4,6])$ is congruent to zero modulo 2 , we obtain $a_{1}+a_{3} \equiv 0 \bmod 2$. Due to 
the linear independence of $a, b, c$, we have $a_{2} \equiv 1$ and $b_{1}+b_{3} \equiv 1 \bmod 2$. Besides, since the coefficients of $X^{3}$ and $Z X^{2}$ in $\phi([3,5,7])$ are congruent modulo 2, we have $a_{1} \equiv 0 \bmod 2$, so $a \equiv(0,1,0) \bmod 2$. Then the coefficient of $X^{3}$ in

$$
\begin{aligned}
\phi([2,4,6]) & \equiv Y\left(X+\left(b_{1}+b_{2}\right) Y\right)\left(b_{1} Y+Z\right) \\
& \equiv\left(0, b_{1}\left(b_{1}+b_{2}\right), 0,0, b_{1}, b_{2}, 1,0,0,1\right) \bmod 2
\end{aligned}
$$

is zero, so the coefficients of $X Y^{2}$ and $X Y Z$ are congruent modulo 2. Hence we have $b_{1} \equiv 1 \bmod 2$, and then $b_{3} \equiv 0 \bmod 2$. However,

$$
\begin{aligned}
\phi([2,5,7]) & \equiv Y\left(X+b_{2} Y+Z\right)\left(X+b_{2} Z\right) \\
& \equiv\left(0, b_{2}, 0,1,0, b_{2}, 0,0,0,1\right) \bmod 2
\end{aligned}
$$

is not a linear combination of $[2,4,6],[2,4,7],[2,5,7]$ and $[3,5,7]$.

- If $c_{1} \equiv 0$ and $c_{2} \equiv 1 \bmod 2$, the second and fourth components of the left-hand side of (15) can not be congruent to zero modulo 2 simultaneously.

- If $c_{1} \equiv c_{2} \equiv 1 \bmod 2$, due to (15), we have $c_{3} \equiv 1 \bmod 2$. From the coefficient of $Z^{2} X$ in $\phi([3,5,7])$, we obtain $a_{2} \equiv a_{3} \bmod 2$. Then $a_{1} \not \equiv a_{2} \bmod 2$ due to the linear independence of $a$ and $c$. Similarly, from the coefficients of $X^{3}$ and $Z X^{2}$ in $\phi([2,4,6])$ and $\phi([2,4,7])$, we obtain $a_{1} \equiv a_{2} \equiv 1 \bmod 2$. This is a contradiction.

- If $c_{1} \equiv c_{2} \equiv 0 \bmod 2$, then we have $c_{3} \equiv 1 \bmod 2$. From the coefficient of $Z^{2} X$ in $\phi([2,5,7])$, we obtain $a_{2} \equiv 0 \bmod 2$. Besides, from $\operatorname{det} \phi \equiv 1 \bmod 2$, we obtain $a_{1} \equiv b_{2} \equiv 1 \bmod 2$. Comparing the coefficients of $X^{3}$ and $Z X^{2}$ in $\phi([2,4,6])$, we obtain $a_{3} \equiv 0 \bmod 2$. Then the coefficient of $X^{3}$ in

$$
\begin{aligned}
\phi([2,4,7]) & \equiv\left(X+\left(b_{1}+b_{3}\right) Y+Z\right)\left(X+\left(b_{1}+1\right) Y\right) Y \\
& \equiv\left(0,\left(b_{1}+b_{3}\right)\left(b_{1}+1\right), 0,1, b_{3}+1, b_{1}+1,0,0,0,1\right) \bmod 2
\end{aligned}
$$

is zero, so the coefficients of $X Y^{2}$ and $X Y Z$ are congruent. Hence $b_{3} \equiv 0 \bmod 2$, and since $\phi([2,4,7])$ needs to be congruent to $(0,0,0,1,1,1,0,0,0,1)$ modulo 2 , we obtain $b_{1} \equiv 0 \bmod 2$.

By Lemma 8.5, $\left(x c_{1}+y c_{2}+c_{3}\right)\left(m c_{1}+n c_{2}+c_{3}\right) \equiv 1 \bmod 2$. Moreover, since $q \equiv 1 \bmod 2$, we have $c_{1}+p c_{2}+q c_{3} \equiv 1 \bmod 2$. Hence we obtain the following corollary from (15). 
Comparing the coefficients of $X^{3}$ and $Z^{2} X$ in $\phi([2,4,6]), \phi([2,4,7]), \phi([2,5,7])$ and $\phi([3,5,7])$, we obtain

$$
\begin{gathered}
q^{\prime} t^{\prime}\left(\begin{array}{c}
\left(a_{1}+p a_{2}+q a_{3}\right)\left(a_{1}+s a_{2}+t a_{3}\right) a_{1} \\
\left(a_{1}+p a_{2}+q a_{3}\right)\left(a_{1}+s a_{2}+t a_{3}\right) a_{2} \\
\left(a_{1}+p a_{2}+q a_{3}\right)\left(m a_{1}+n a_{2}+a_{3}\right) a_{2} \\
\left(x a_{1}+y a_{2}+a_{3}\right)\left(m a_{1}+n a_{2}+a_{3}\right) a_{2}
\end{array}\right) \\
=\left(\begin{array}{c}
\left(c_{1}+q c_{3}\right)\left\{\left(c_{1}+t c_{3}\right) a_{1}+\left(a_{1}+s a_{2}+t a_{3}\right) c_{1}\right\} \\
\left(c_{1}+q c_{3}\right)\left(c_{1}+t c_{3}\right) a_{2} \\
\left(c_{1}+q c_{3}\right)\left(m c_{1}+c_{3}\right) a_{2} \\
\left(x c_{1}+c_{3}\right)\left(m c_{1}+c_{3}\right) a_{2}
\end{array}\right) .
\end{gathered}
$$

If we assume $a_{2} \neq 0$, from (16) we obtain $q^{\prime} t^{\prime}\left(x a_{1}+y a_{2}+a_{3}\right)\left(m a_{1}+n a_{2}+a_{3}\right)=$ $\left(x c_{1}+c_{3}\right)\left(m c_{1}+c_{3}\right)$. The left hand side of this equation is even but the right hand side is odd. Hence we have the following lemma.

\section{Lemma 8.7 $\quad a_{2}=0$}

By Corollary 8.6 and Lemma 8.7, we obtain the following proposition.

Proposition 8.8 $\quad\left(a_{2}, b_{2}, c_{2}\right)= \pm(0,1,0)$

Considering $-\phi$ if necessary, we can assume $b_{2}=1$.

First, we consider the case $c_{1} \neq 0$. From (15), we have $c_{1}+t c_{3}=0$. Hence $t \neq 0$ (note that, in view of the list of characteristic matrices, this means $t=2)$ and $c=c_{3}(-2,0,1)$, where $c_{3}= \pm 1$. From (16), we have

$$
q^{\prime} t^{\prime}\left(a_{1}+q a_{3}\right)\left(a_{1}+2 a_{3}\right) a_{1}=-2(q-2)\left(a_{1}+2 a_{3}\right) .
$$

Since $a_{1}+2 a_{3} \neq 0$ by Lemma 8.5 , this equation reduces to

$$
q^{\prime} t^{\prime}\left(a_{1}+q a_{3}\right) a_{1}=-2(q-2) .
$$

Moreover, since the right hand of this equation is not zero, we have $t^{\prime}=t=2$ and

$$
q^{\prime}\left(a_{1}+q a_{3}\right) a_{1}=2-q .
$$

Comparing the coefficients of $X^{3}$ and $Z X^{2}$ in $\phi([2,4,6])$, we obtain

$$
\left(q^{\prime}+2\right)\left(a_{1}+q a_{3}\right)\left(a_{1}+2 a_{3}\right) a_{1}=\left(a_{1}+2 a_{3}\right)\left\{\left(a_{1}+q a_{3}\right)\left(-2 c_{3}\right)+(q-2) c_{3} a_{1}\right\} .
$$

Since $a_{1}+2 a_{3} \neq 0$, this equation can be shortened to

$$
\left(q^{\prime}+2\right)\left(a_{1}+q a_{3}\right) a_{1}=\left\{-2\left(a_{1}+q a_{3}\right)+(q-2) a_{1}\right\} c_{3} .
$$


Since $\operatorname{det} \phi= \pm 1$, we obtain $c_{3}\left(a_{1}+2 a_{3}\right)= \pm 1$. Putting $\epsilon=c_{3} \operatorname{det} \phi$, we have $a_{1}+2 a_{3}=\epsilon$. Then (17) is rewritten as

$$
\left(\epsilon+(q-2) a_{3}\right)\left(\epsilon-2 a_{3}\right) q^{\prime}=2-q .
$$

- If $q=1$ or $3, \epsilon-2 a_{3}$ divides 1 . Since $a_{3}$ is even, we obtain $a_{3}=0$.

- If $q=-1, \epsilon-3 a_{3}$ divides 3 . Hence we obtain $a_{3}=0$.

In any case, we obtain $a_{3}=0$, implying that $q^{\prime}=2-q$. From (18), we have $q^{\prime}+2=-\left(q^{\prime}+2\right) a_{1} c_{3}$, namely, $a_{1} c_{3}=-1$. Thus we obtain the following lemma.

Lemma 8.9 If $c_{1} \neq 0$, we have $t^{\prime}=t=2, q^{\prime}=2-q$ and

$$
\phi= \pm\left(\begin{array}{ccc}
a_{1} & b_{1} & 2 a_{1} \\
0 & 1 & 0 \\
0 & b_{3} & -a_{1}
\end{array}\right) .
$$

Then we consider the cases $\left(q, q^{\prime}\right)=(3,-1),\left(q, q^{\prime}\right)=(-1,3)$ and $\left(q, q^{\prime}\right)=(1,1)$ separately.

Case 1 If $q=3$ and $q^{\prime}=-1$, then $\lambda=\left(\lambda_{28} ; 0,0\right)$ and $\lambda^{\prime}=\left(\lambda_{26} ; 0,0\right),\left(\lambda_{27} ; 0,0\right)$. The matrix (12) for $\lambda^{\prime}$ reduces to

$$
\left(\begin{array}{rrrccrrrcc}
1 & 0 & 0 & 1 & 0 & 0 & 0 & -2 & 1 & -1 \\
0 & 0 & 0 & 1 & 1 & -1 & -2 & 0 & 0 & 1 \\
0 & 0 & 0 & m^{\prime} & 1 & -1 & -1 & 0 & 0 & 1-m^{\prime} \\
0 & 1 & 0 & m^{\prime} & m^{\prime}+1 & 2 & 1 & 0 & 0 & 1+m^{\prime}
\end{array}\right) .
$$

Since the coefficient of $Y^{3}$ in $\phi\left([2,4,6]-b_{1}[2,4,7]\right)$ is zero, the coefficients of $X Y^{2}$ and $Y^{2} Z$ in $\phi\left([2,4,6]-b_{1}[2,4,7]\right)$ are negatives of each other. Then we have

$$
a_{1}\left(b_{1}+3 b_{3}+2\right)\left(b_{1}+2 b_{3}+1\right)=-2 a_{1}\left(b_{1}+3 b_{3}+2\right)\left(b_{1}+2 b_{3}+1\right) .
$$

Since $a_{1}, b_{1}+2 b_{3}+1 \equiv 1 \bmod 2$, this equation reduces to $b_{1}+3 b_{3}+2=0$. Then $\phi\left([2,4,6]-b_{1}[2,4,7]\right)$

$$
\begin{aligned}
& =(X-Z)\left(a_{1} X-\left(b_{3}+1\right) Y\right)(X+2 Z) \\
& =\left(a_{1}, 0,0,-b_{3}-1,0,0,2\left(b_{3}+1\right),-2 a_{1}, a_{1},-b_{3}-1\right) \\
& \equiv\left(0,0,0,-a_{1}-b_{3}-1,0,0,2\left(b_{3}+1\right), 0,0, a_{1}-b_{3}-1\right) \bmod \mathcal{I}_{\lambda^{\prime}}^{6} .
\end{aligned}
$$

Hence there exists an integer $\alpha$ such that

$$
\alpha\left(1-m^{\prime}\right)=-a_{1}-b_{3}-1, \quad-\alpha=2\left(b_{3}+1\right), \quad \alpha m^{\prime}=a_{1}-b_{3}-1 .
$$


We obtain $\left(b_{3}+1\right)\left(1-2 m^{\prime}\right)=a_{1}= \pm 1$ from these equations. Then we have $m^{\prime}=0$, $b_{3}=a_{1}-1$. Therefore we see that

$$
\phi=\left(\begin{array}{rrr}
1 & -2 & 2 \\
0 & 1 & 0 \\
0 & 0 & -1
\end{array}\right) \quad \text { or } \quad\left(\begin{array}{rrr}
-1 & 4 & -2 \\
0 & 1 & 0 \\
0 & -2 & 1
\end{array}\right)
$$

In each case,

$$
\phi([2,5,7])=(X-Z)(Y-Z) Y=(0,0,0,0,1,-1,1,0,0,-1) \notin \mathcal{I}_{\lambda^{\prime}}^{6} .
$$

Hence we see that there exists no graded ring isomorphism from $H^{*}(\lambda)$ to $H^{*}\left(\lambda^{\prime}\right)$ in this case.

Case 2 If $q=-1$ and $q^{\prime}=3$, from the conclusion of the previous case, we see that there exists no graded ring isomorphism from $H^{*}(\lambda)$ to $H^{*}\left(\lambda^{\prime}\right)$.

Case 3 If $q=q^{\prime}=1$, then we can assume $\lambda, \lambda^{\prime}=\left(\lambda_{19} ; 0,0\right),\left(\lambda_{21} ; 0,0\right),\left(\lambda_{23} ; 0,0\right)$, $\left(\lambda_{25} ; 0,0\right)$. In a similar way to Case 1 , we can show that all graded ring automorphisms that map $\mathcal{I}_{\lambda}^{6}$ into $\mathcal{I}_{\lambda^{\prime}}^{6}$ and such that $c_{1} \neq 0$ are in the illustration below:

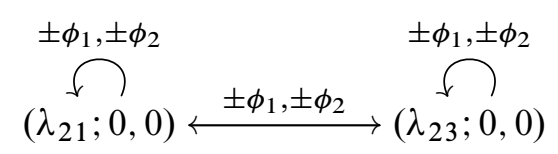

Here

$$
\phi_{1}=\left(\begin{array}{rrr}
1 & 2 & 2 \\
0 & 1 & 0 \\
0 & -2 & -1
\end{array}\right) \quad \text { and } \quad \phi_{2}=\left(\begin{array}{rrr}
-1 & 0 & -2 \\
0 & 1 & 0 \\
0 & 0 & 1
\end{array}\right)
$$

Next, in the case that $c_{1}=0$, by the same method as above, we can show that all nontrivial graded ring automorphisms that map $\mathcal{I}_{\lambda}^{6}$ into $\mathcal{I}_{\lambda^{\prime}}^{6}$ and such that $c_{1}=0$ are in the illustration below:

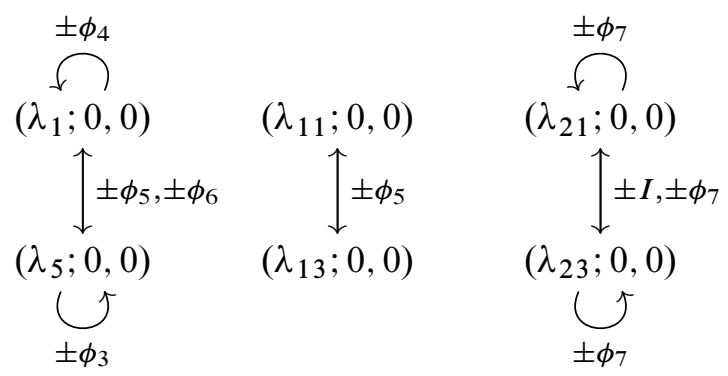


Here $\phi_{3}, \ldots, \phi_{7}$ denote the matrices

$$
\begin{array}{ll}
\phi_{3}=\left(\begin{array}{rrr}
-1 & -2 & 0 \\
0 & 1 & 0 \\
0 & 2 & -1
\end{array}\right), \quad \phi_{4}=\left(\begin{array}{rrr}
-1 & -2 & 0 \\
0 & 1 & 0 \\
0 & -2 & -1
\end{array}\right), & \\
\phi_{5}=\left(\begin{array}{rrr}
1 & 0 & 0 \\
0 & 1 & 0 \\
0 & 0 & -1
\end{array}\right), \quad \phi_{6}=\left(\begin{array}{rrr}
-1 & -2 & 0 \\
0 & 1 & 0 \\
0 & -2 & 1
\end{array}\right), \quad \phi_{7}=\left(\begin{array}{rrr}
-1 & 2 & 0 \\
0 & 1 & 0 \\
0 & -2 & -1
\end{array}\right) .
\end{array}
$$

Now we have all graded ring automorphisms of $\mathbb{Z}[X, Y, Z]$ which map $\mathcal{I}_{\xi}^{6}$ into $\mathcal{I}_{\xi^{\prime}}^{6}$ with respect to some characteristic matrices $\xi$ and $\xi^{\prime}$ on $C^{5}(8)^{*}$. In fact, none of these automorphisms maps $\mathcal{I}_{\xi}^{8}$ into $\mathcal{I}_{\xi^{\prime}}$. One can verify it by means of a computer-assisted calculation. Thus we have the topological classification of quasitoric manifolds over $C^{5}(8)^{*}$.

Theorem 8.10 There are exactly forty-six quasitoric manifolds over $C^{5}(8)^{*}$ up to homeomorphism, and they are distinguished by their cohomology rings.

Acknowledgements The author is deeply grateful to Daisuke Kishimoto for his support and valuable advice, and also appreciates the comments of the referee, which helped to improve the paper.

\section{References}

[1] V M Buchstaber, T E Panov, Torus actions and their applications in topology and combinatorics, University Lecture Series 24, Amer. Math. Soc. (2002) MR1897064

[2] S Choi, M Masuda, D Y Suh, Rigidity problems in toric topology: A survey, Tr. Mat. Inst. Steklova 275 (2011) 188-201 MR2962979 Also in Proc. Steklov Inst. Math. 275 (2011), 177-190

[3] S Choi, S Park, DY Suh, Topological classification of quasitoric manifolds with second Betti number 2, Pacific J. Math. 256 (2012) 19-49 MR2928539

[4] M Davis, Smooth G-manifolds as collections of fiber bundles, Pacific J. Math. 77 (1978) 315-363 MR510928

[5] M W Davis, T Januszkiewicz, Convex polytopes, Coxeter orbifolds and torus actions, Duke Math. J. 62 (1991) 417-451 MR1104531

[6] PE Jupp, Classification of certain 6-manifolds, Proc. Cambridge Philos. Soc. 73 (1973) 293-300 MR0314074

[7] P Orlik, F Raymond, Actions of the torus on 4-manifolds, I, Trans. Amer. Math. Soc. 152 (1970) 531-559 MR0268911 
[8] G M Ziegler, Lectures on polytopes, Graduate Texts in Mathematics 152, Springer, New York (1995) MR1311028

Department of Mathematics, Faculty of Science, Kyoto University Sakyo-ku, Kyoto 606-8502, Japan

s.hasui@math.kyoto-u.ac.jp

Received: 24 November $2013 \quad$ Revised: 27 April 2014 
